Homology, Homotopy and Applications, vol.18(2), 2016, pp.107-132

\title{
THE GHOST LENGTH AND DUALITY ON THE CHAIN AND COCHAIN TYPE LEVELS
}

\author{
KATSUHIKO KURIBAYASHI
}

(communicated by J.P.C. Greenlees)

\begin{abstract}
We establish equalities between cochain and chain type levels of maps by making use of exact functors which connect appropriate derived and coderived categories. Relevant conditions for levels of maps to be finite are extracted from the equalities which we call duality on the levels. Moreover, we give a lower bound of the cochain type level of the diagonal map on the classifying space of a Lie group by considering the ghostness of a shriek map which appears in derived string topology. A variant of Koszul duality for a differential graded algebra is also discussed.
\end{abstract}

\section{Introduction}

This work is a sequel to previous one $[32,33]$ in which new topological invariants have been studied.

In [2], Avramov, Buchweitz, Iyengar and Miller introduced a numerical invariant of an objects in a triangulated category, which is called the level. The invariant counts the number of steps to build the given object out of some fixed object via triangles. It

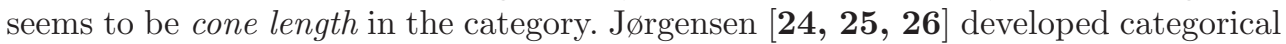
representation theory of spaces employing the singular (co)chain complexes of spaces. In the context of such work, the cochain and chain type levels of maps between topological spaces have been defined and studied in $[32,33]$.

The cochain type level of a map $\alpha: Y \rightarrow X$ indeed provides a lower bound on the number of spherical fibrations which describe a factorization of $\alpha$ in a relevant sense; see [32, Proposition 2.11]. On the other hand, the chain type level of the identity map on a space $Y$ gives an upper bound of the L.-S. category of $Y$ in rational case; see [33, Corollary 2.9]. The L.-S. category is also considered a homotopy invariant counting the number of cofibrations which construct a given space. Therefore, it is natural to anticipate that the levels of maps inherit duality between fibrations and cofibrations, namely Eckmann-Hilton duality. For example, one might expect that chain and cochain type levels fit into appropriate equalities, which we may call duality on the levels.

The author was supported in part by Grant-in-Aid for Scientific Research (B) JP 25287008 from Japan Society for the Promotion of Science.

Received April 1, 2015, revised November 9, 2015, January 12, 2016; published on August 10, 2016. 2010 Mathematics Subject Classification: 16E45, 18E30, 55R20, 13 D07.

Key words and phrases: level, differential graded algebra, triangulated category, Koszul duality.

Article available at http://dx.doi.org/10.4310/HHA.2016.v18.n2.a6

Copyright (C) 2016, International Press. Permission to copy for private use granted. 
In this article, we establish such equalities between these two kinds of levels; see Theorem 2.4 below. One of the highlights in getting the result is that we make use of a variant of Koszul duality for differential graded algebras which is given by considering exact functors between certain derived and coderived categories; see $[\mathbf{2 1}, \mathbf{2 8}, \mathbf{3 8}, \mathbf{4 6}]$ for Koszul duality. In fact, Theorem 3.3 below describes such a variant. We can take the singular chains of a space as a coalgebra in Theorem 3.3. In consequence, the commutative diagrams of categories in the theorem give duality of the levels in Theorem 2.4.

Let $X$ be a space and $L X$ the free loop space, namely the space of all continuous maps from the circle $S^{1}$ to $X$ with compact-open topology. String topology initiated by the fascinating paper of Chas and Sullivan [8] describes a rich structure in the homology of the free loop space $L M$ of a closed oriented manifold $M$. A basic one of the string operations is the so-called loop product on the shifted homology $H_{*+\operatorname{dim} M}(L M)$. The key to defining these operations is to construct a shriek map (an Umkehr map or a wrong way map) associated with the diagonal map on $M$.

Félix and Thomas [17] generalized the construction of shriek maps on manifolds to that on Gorenstein spaces. This enables us to develop string topology in appropriate derived categories; see $[\mathbf{3 6}, \mathbf{3 7}]$ for torsion and extension functor descriptions of loop (co)products and their applications. It is important to mention that the class of Gorenstein spaces contains the classifying spaces of connected Lie groups, Borel constructions more general, Poincaré duality spaces and hence closed oriented manifolds; see $[12,13,44]$.

Let $B G$ be the classifying space of a connected Lie group $G$. In [9], Chataur and Menichi showed that the homology $H_{*}(L B G ; \mathbb{K})$ with coefficients in a field $\mathbb{K}$ carries the structure of homological conformal field theory (HCFT). The integration along the fibre of a Borel fibration plays a crucial role in defining the HCFT operations. In a derived categorical setting, the integration is considered the homomorphism induced by a shriek map on the derived category $\mathrm{D}\left(C^{*}(B G \times B G)\right)$ of differential graded modules (DG modules) over the cochain algebra $C^{*}(B G \times B G)$ with coefficients in $\mathbb{K}$; see $[\mathbf{1 7}$, Theorems 5 and 13$]$.

Another aim of this article is to consider behavior of such shriek maps in the derived category $\mathrm{D}\left(C^{*}(B G \times B G)\right)$, more generally in $\mathrm{D}\left(C^{*}\left(B G^{\times n}\right)\right)$. In particular, we see that non-triviality of a shriek map associated with the diagonal map on $B G$ in $\mathrm{D}\left(C^{*}\left(B G^{\times n}\right)\right)$ gives a lower bound of the ghost length of $C^{*}(B G)$; see Theorem 2.11 and Remark 5.2. In consequence, a lower bound of the cochain type level of the diagonal map $B G \rightarrow B G^{\times n}$ is obtained; see Proposition 2.12. We mention that the notion of ghosts has been actually introduced by Christensen [10] in a more general framework.

We conclude this section with comments on topics related to the invariant level. Our attempt in $[\mathbf{3 2}, \mathbf{3 3}]$ and this paper is closely related to the work in $[\mathbf{5}, \mathbf{1 1}, \mathbf{4 7}, \mathbf{4 9}]$. Indeed, the dimension $\operatorname{dim} \mathcal{T}$ of a triangulated category $\mathcal{T}$, which is introduced by Rouquier [47]; see also [7], is defined by

$$
\operatorname{dim} \mathcal{T}=\inf \left\{d \in \mathbb{N} \mid \operatorname{thick}_{\mathcal{T}}^{d+1}(C)=\mathcal{T} \text { for some object } C \text { in } \mathcal{T}\right\} .
$$

Here $\operatorname{thick}_{\mathcal{T}}^{j}(C)$ denotes the $j$ th thickening which is a subcategory of $\mathcal{T}$ used when defining the level; see Section 2. Thus the dimension gives a global invariant of triangulated categories. 
The results in [5] due to Benson, Iyengar and Krause are concerned with the classification of thick subcategories of a triangulated category. In [11, 2.1 Theorem], Dwyer and Greenlees give an equivalence between categories of torsion and complete modules. Moreover, the result [11, 4.6 Proposition] asserts that torsion modules are chain complexes built from a fixed complex. Then these also clarify global nature of thick or localizing subcategories.

On the other hand, the level considered here captures properties of individual objects, which come from topological spaces via the singular chain and cochain functors; see Remark 2.3 below.

In [12], Dwyer, Greenlees and Iyengar have developed Morita theory in algebraic topology by making use of ring spectra. In particular, the result [12, 3.16 Proposition] describes a necessary and sufficient condition for the level of a map to be finite. Very recently, Mao [39] has introduced a new numerical invariant for DG modules, which is defined by replacing thick subcategories in the definition of the level with localizing ones. The invariant of a bounded below DG module coincides with the ghost length plus one; see [39, Theorem A].

Following [49], the string topology category invented by Blumberg, Cohen and Teleman [6] can be regarded as a full subcategory of one of the derived categories that we deal with in this paper. Then we can expect that machinery used in order to investigate the invariant level is applicable to the study of the string topology category; see Remark 3.9 for such expectation. It is worth noting that the recent result [49, Theorem 1.2] due to Shamir, which is concerned with the string topology category, is deduced by relying on the results in $[\mathbf{5}, \mathbf{1 1}]$ cited above.

\section{Results}

To describe our results more precisely, we first recall from [2, Section 2] the definition of the level of an object in a triangulated category $\mathcal{T}$. We say that a subcategory of $\mathcal{T}$ is strict if it is closed under isomorphisms in $\mathcal{T}$.

For a given object $C$ in $\mathcal{T}$, we define the 0th thickening by $\operatorname{thick}_{\mathcal{T}}^{0}(C)=\{0\}$ and $\operatorname{thick}_{\mathcal{T}}^{1}(C)$ to be the smallest strict full subcategory which contains $C$ and is closed under taking finite coproducts, retracts and all shifts. Moreover for $n>1$ define inductively the $n$th thickening $\operatorname{thick}_{\mathcal{T}}^{n}(C)$ by the smallest strict full subcategory of $\mathcal{T}$ which is closed under retracts and contains objects $M$ admitting a distinguished triangle $M_{1} \rightarrow M \rightarrow M_{2} \rightarrow \Sigma M_{1}$ in $\mathcal{T}$ for which $M_{1}$ and $M_{2}$ are in thick ${ }_{\mathcal{T}}^{n-1}(C)$ and $\operatorname{thick}_{\mathcal{T}}^{1}(C)$, respectively. A triangulated subcategory $\mathcal{C}$ of $\mathcal{T}$ is said to be thick if it is closed under taking retracts. Then the thickenings provide a filtration of the smallest thick subcategory thick $\mathcal{T}(C)$ of $\mathcal{T}$ containing the object $C$ :

$$
\{0\}=\operatorname{thick}_{\mathcal{T}}^{0}(C) \subset \cdots \subset \operatorname{thick}_{\mathcal{T}}^{n}(C) \subset \cdots \subset \cup_{n \geqslant 0} \operatorname{thick}_{\mathcal{T}}^{n}(C)=\operatorname{thick}_{\mathcal{T}}(C) .
$$

For an object $M$ in $\mathcal{T}$, we define a numerical invariant $\operatorname{level}_{\mathcal{T}}^{C}(M)$, which is called the $C$-level of $M$, by

$$
\operatorname{level}_{\mathcal{T}}^{C}(M):=\inf \left\{n \in \mathbb{N} \mid M \in \operatorname{thick}_{\mathcal{T}}^{n}(C)\right\} .
$$

It turns out that the $C$-level of an object $M$ in $\mathcal{T}$ counts the number of steps required to build $M$ out of the object $C$ via triangles. For more details and general features of the level, we refer the reader to [2, Sections 2 and 3]. 
Let $\mathbb{K}$ be a field of arbitrary characteristic and $R$ a DG (that is, differential graded) algebra over $\mathbb{K}$. Let $\mathrm{D}(R)$ denote the derived category of $\mathrm{DG}$ right $R$-modules. Observe that the category $\mathrm{D}(R)$ comes equipped with the structure of a triangulated category [27], in particular with the shift functor $\Sigma$ defined by $(\Sigma M)^{n}=M^{n+1}$.

We here recall from $[\mathbf{3 2}]$ and $[\mathbf{3 3}]$ two numerical topological invariants defined by the level in a triangulated category $\mathrm{D}(R)$. Unless otherwise explicitly stated, it is assumed that a space has the homotopy type of a connected CW complex whose cohomology with coefficients in the underlying field is locally finite.

Let $B$ be a space and $\mathcal{T O P}_{B}$ the category of maps with the target $B$; that is, an object of $\mathcal{T O P}_{B}$ is a map $f: X \rightarrow B$ and a morphism form $f: X \rightarrow B$ to $g: X \rightarrow B$ is a map $\alpha: X \rightarrow Y$ which satisfies the condition that $f=g \circ \alpha$. For any object $f: X \rightarrow B$, the normalized singular cochain $C^{*}(X ; \mathbb{K})$ with coefficients in $\mathbb{K}$ is regarded as a DG right module over the cochain algebra $C^{*}(B ; \mathbb{K})$ via the induced map $C^{*}(f): C^{*}(B ; \mathbb{K}) \rightarrow C^{*}(X ; \mathbb{K})$. Thus the cochain functor gives rise to a contravariant functor from the category $\mathcal{T O P} \mathcal{P}_{B}$ to the triangulated category $\mathrm{D}\left(C^{*}(B ; \mathbb{K})\right)$ :

$$
C^{*}(s(-) ; \mathbb{K}): \mathcal{T O} \mathcal{P}_{B} \rightarrow \mathrm{D}\left(C^{*}(B ; \mathbb{K})\right),
$$

where $s(f)$ denotes the source of an object $f$ in $\mathcal{T O} \mathcal{P}_{B}$.

Definition 2.1. Let $f$ be an object of $\mathcal{T O P} \mathcal{P}_{B}$. The cochain type level of the map $f$ is defined by the $C^{*}(B ; \mathbb{K})$-level of the DG module $C^{*}(s(f) ; \mathbb{K})$, namely

$$
\operatorname{level}_{\mathrm{D}\left(C^{*}(B ; \mathbb{K})\right)}^{C^{*}(B ; \mathbb{K})}\left(C^{*}(s(f) ; \mathbb{K})\right) .
$$

Let $F_{f}$ be the homotopy fibre of a map $f: X \rightarrow B$. The Moore loop space $\Omega B$ acts on the space $F_{f}$ by the holonomy action. Thus the normalized chain complex $C_{*}\left(F_{f} ; \mathbb{K}\right)$ is a DG module over the chain algebra $C_{*}(\Omega B ; \mathbb{K})$. The normalized singular chain and the homotopy fibre construction enable us to obtain a covariant functor

$$
C_{*}\left(F_{(-)} ; \mathbb{K}\right): \mathcal{T O} \mathcal{P}_{B} \rightarrow \mathrm{D}\left(C_{*}(\Omega B ; \mathbb{K})\right)
$$

from the category $\mathcal{T O} \mathcal{P}_{B}$ to the triangulated category $\mathrm{D}\left(C_{*}(\Omega B ; \mathbb{K})\right)$.

Definition 2.2. Let $f$ be an object of $\mathcal{T O} \mathcal{P}_{B}$. The chain type level of the map $f$ is defined by the $C_{*}(\Omega B ; \mathbb{K})$-level of the DG module $C_{*}\left(F_{f} ; \mathbb{K}\right)$, namely

$$
\operatorname{level}_{\mathrm{D}\left(C_{*}(\Omega B ; \mathbb{K})\right)}^{C_{*}(\Omega B ; \mathbb{K})}\left(C_{*}\left(F_{f} ; \mathbb{K}\right)\right) .
$$

More generally, we call the levels of objects in $\mathrm{D}\left(C_{*}(\Omega B ; \mathbb{K})\right)$ and in $\mathrm{D}\left(C^{*}(B ; \mathbb{K})\right)$ the chain type levels and the cochain type levels, respectively. In what follows, the coefficients in the singular (co)chain complex and their homology are often omitted if the context makes them clear.

Remark 2.3. Let $\mathcal{T}^{c}$ be the full subcategory of the triangulated category $\mathcal{T}=\mathrm{D}(A)$ consisting of compact objects, where $A=C^{*}\left(S^{d} ; \mathbb{K}\right)$. The result [48, Proposition 6.6] implies that for any $i \in \mathbb{N}$, there exists an indecomposable object $Z_{i}$ in $\mathcal{T}^{c}$ such that level $_{\mathcal{T}}^{A} Z_{i}=\operatorname{level}_{\mathcal{T}^{c}}^{A} Z_{i}=i+1$. On the other hand, $\operatorname{dim} \mathcal{T}^{c}=\infty$. In fact, if $\operatorname{dim} \mathcal{T}^{c}=$ $l<\infty$, then there is an object $C$ in $\mathcal{T}^{c}$ such that $\mathcal{T}^{c}=\operatorname{thick}_{\mathcal{T}^{c}}^{l+1}(C)$. This yields that level $\mathrm{T}_{\mathcal{T}^{c}}^{C} M \leqslant l+1$ for any object $M \in \mathcal{T}^{c}$. Since $C$ is compact, it follows from [27, 
Theorem 5.3] that level ${ }_{\mathcal{T}}^{A} C=n$ for some $n$. Then a triangular inequality (Lemma 4.1) implies that

$$
\operatorname{level}_{\mathcal{T}}^{A} M \leqslant \operatorname{level}_{\mathcal{T}}^{A} C \cdot \operatorname{level}_{\mathcal{T}}^{C} M \leqslant n\left(\operatorname{dim} \mathcal{T}^{c}+1\right)
$$

for any $M$ in $\mathcal{T}^{c}$. As mentioned above, we have an indecomposable object $Z$ in $\mathcal{T}^{c}$ with level ${ }_{\mathcal{T}}^{A} Z>n\left(\operatorname{dim} \mathcal{T}^{c}+1\right)$, which is a contradiction.

One of our main theorems reveals a remarkable relationship between the two kinds of levels.

Theorem 2.4. Let $B$ be a simply-connected space and $f: X \rightarrow B$ an object in $\mathcal{T O P}_{B}$. Then one has (in) equalities

(1) $\operatorname{dim} H_{*}(X ; \mathbb{K}) \geqslant \operatorname{level}_{\mathrm{D}\left(C_{*}(\Omega B)\right)}^{C_{*}(\Omega B)}\left(C_{*}\left(F_{f}\right)\right)=\operatorname{level}_{\mathrm{D}\left(C^{*}(B)\right)}^{\mathbb{K}}\left(C^{*}(X)\right)$ and

(2) $\operatorname{dim} H^{*}\left(F_{f} ; \mathbb{K}\right) \geqslant \operatorname{level}_{\mathrm{D}\left(C^{*}(B)\right)}^{C^{*}(B)}\left(C^{*}(X)\right)=\operatorname{level}_{\mathrm{D}\left(C_{*}(\Omega B)\right)}^{\mathbb{K}}\left(C_{*}\left(F_{f}\right)\right)$.

As mentioned in the Introduction, the theorem is deduced from a correspondence between the triangulated categories $\mathrm{D}\left(C_{*}(\Omega B)\right)$ and $\mathrm{D}\left(C^{*}(B)\right)$, which is a variant of Koszul duality for DG algebras; see Theorem 3.3, Proposition 3.6 and Theorem A.4. More precisely, we deduce the results by means of exact functors between the triangulated categories which are compatible with the covariant functor $C_{*}\left(F_{(-)}\right)$and the contravariant functor $C^{*}(s(-))$. These would allow us to call the equalities in Theorem 2.4 duality on the (co)chain type levels. Algebraic versions of the equalities above deserve mention. They appear in Remark 3.8.

We here describe another evidence that the equalities in Theorem 2.4, which are topological versions, exhibit the duality. By definition, the homotopy fibre $F_{f}$ for a given map $f: X \rightarrow B$ fits into a sequence

$$
\Omega B \stackrel{i}{\longrightarrow} F_{f} \stackrel{p}{\longrightarrow} X \stackrel{f}{\longrightarrow} B
$$

in which $p$ is a fibration with $\Omega B$ the fibre. We observe that the maps $i$ and $f$ give the chain $C_{*}\left(F_{f}\right)$ and the cochain $C^{*}(X)$ a $C_{*}(\Omega B)$-module structure and a $C^{*}(B)$ module structure, respectively. Since the map $p$ connects those maps $i$ and $f$, it seems that (in)equalities in Theorem 2.4 reflect homological duality of the fibration in some sense. In fact, the Eilenberg-Moore type quasi-isomorphism relative to a fibration $[\mathbf{1 4}, \mathbf{1 5}]$ is an important ingredient for proving the main theorem; see Proposition 3.6.

Theorem 2.4 and a triangular inequality on the levels (Lemma 4.1) allow us to compare the chain and cochain type levels of maps.

Proposition 2.5. Under the same assumption as in Theorem 2.4, one has inequalities

$$
\begin{aligned}
\operatorname{level}_{\mathrm{D}\left(C_{*}(\Omega B)\right)}^{C_{*}(\Omega B)}\left(C_{*}\left(F_{f}\right)\right) & \leqslant \operatorname{level}_{\mathrm{D}\left(C_{*}(\Omega B)\right)}^{C_{*}(\Omega B)}(\mathbb{K}) \cdot \operatorname{level}_{\mathrm{D}\left(C^{*}(B)\right)}^{C^{*}(B)}\left(C^{*}(X)\right) \\
& \leqslant \operatorname{dim} H_{*}(B ; \mathbb{K}) \cdot \operatorname{level}_{\mathrm{D}\left(C^{*}(B)\right)}^{C^{*}(B)}\left(C^{*}(X)\right) \quad \text { and } \\
\operatorname{level}_{\mathrm{D}\left(C^{*}(B)\right)}^{C^{*}(B)}\left(C^{*}(X)\right) & \leqslant \operatorname{level}_{\mathrm{D}\left(C^{*}(B)\right)}^{C^{*}(B)}(\mathbb{K}) \cdot \operatorname{level}_{\mathrm{D}\left(C_{*}(\Omega B)\right)}^{C_{*}(\Omega B)}\left(C_{*}\left(F_{f}\right)\right) \\
& \leqslant \operatorname{dim} H^{*}(\Omega B ; \mathbb{K}) \cdot \operatorname{level}_{\mathrm{D}\left(C_{*}(\Omega B)\right)}^{C_{*}(\Omega B)}\left(C_{*}\left(F_{f}\right)\right)
\end{aligned}
$$


As a corollary of Theorem 2.4, we have criteria for the levels of maps to be finite. Let $M$ be an object of the triangulated category $\mathrm{D}(R)$ of DG-modules over a DG algebra $R$. Then it is immediate that $\operatorname{dim} H(M)<\infty$ if $\operatorname{level}_{\mathrm{D}(R)}^{\mathbb{K}} M<\infty$. Thus we have the following result.

Corollary 2.6. Let $f: X \rightarrow B$ be a map with $B$ simply-connected.

(1) level ${ }_{\mathrm{D}\left(C_{*}(\Omega B)\right)}^{C_{*}(\Omega B)}\left(C_{*}\left(F_{f}\right)\right)$ is finite if and only if so is $\operatorname{dim} H_{*}(X ; \mathbb{K})$.

(2) $\operatorname{level}_{\mathrm{D}\left(C^{*}(B)\right)}^{C^{*}(B)}\left(C^{*}(X)\right)$ is finite if and only if so is $\operatorname{dim} H^{*}\left(F_{f} ; \mathbb{K}\right)$.

This corollary is essentially a special case of [18, Proposition 2.3]; see also [2, Theorem 4.8] for other equivalence conditions for the level to be finite.

Let $X$ be a simply-connected rational space. The result [33, Corollary 2.9] states that

$$
\text { cat } X \leqslant \operatorname{level}_{\mathrm{D}\left(C_{*}(\Omega X)\right)}^{C_{*}(\Omega X)} \mathbb{Q}-1,
$$

where cat $X$ stands for the L.-S. category of $X$. Moreover, a simple calculation in $[\mathbf{3 3}$ Example 6.4] enables us to conclude that if $X$ is a simply-connected rational $\mathrm{H}$-space with $\operatorname{dim} H^{*}(X ; \mathbb{Q})<\infty$, then the above inequality turns out to be the equality. On the other hand, the inequality can be strict as we will see below.

Example 2.7. Let $X$ be an infinite wedge of spheres of the form $\bigvee_{\alpha} S^{n_{\alpha}}$. Then $\operatorname{cat} X_{\mathbb{Q}}=\operatorname{cat} X=1$. By applying Corollary 2.6(1) to the case where $i d_{X}: X \rightarrow X$, we see that level ${ }_{\mathrm{D}\left(C_{*}(\Omega X)\right)}^{C_{*}(\Omega X)} \mathbb{Q}=\infty$. In fact, $H_{*}(X ; \mathbb{Q})$ is of infinite dimension.

The following proposition, which is derived from Corollary 2.6(2) and the totally fibred square construction [45, Section 3], is of interest to us. Indeed, the result suggests that the study of the levels for maps contributes to determining the homotopy types of spaces.

Proposition 2.8. Let $\pi: X \rightarrow B$ be a map between simply-connected spaces with a right homotopy inverse $s: B \rightarrow X$. We regard the map $\pi$ and $s$ as objects in $\mathcal{T O P} \mathcal{P}_{B}$ and $\mathcal{T O P} \mathcal{P}_{X}$, respectively. Then both of levels level ${ }_{\mathrm{D}\left(C^{*}(B ; \mathbb{K})\right)}^{C^{*}(B \mathbb{K})} C^{*}(X ; \mathbb{K})$ and level $_{\mathrm{D}\left(C^{*}(X ; \mathbb{K})\right)}^{C^{*}(X \mathbb{K})} C^{*}(B ; \mathbb{K})$ are finite if and only if $H^{*}\left(F_{\pi} ; \mathbb{K}\right)=\mathbb{K}$. In particular, the both of the two levels with coefficients in $\mathbb{Z} / p$ are finite if and only if $\pi: X \rightarrow B$ is a homotopy equivalence after $p$-completion.

Let $q: X \rightarrow B$ be a trivial fibration with $H^{*}\left(F_{q} ; \mathbb{K}\right)$ finite dimensional. Then the $C^{*}(B ; \mathbb{K})$-level of $C^{*}(X ; \mathbb{K})$ in $\mathrm{D}\left(C^{*}(B ; \mathbb{K})\right)$ is just one for any field $\mathbb{K}$ in general. In fact, we see that $C^{*}(X ; \mathbb{K}) \cong C^{*}(B ; \mathbb{K}) \otimes H^{*}\left(F_{q} ; \mathbb{K}\right)$ in $\mathrm{D}\left(C^{*}(B ; \mathbb{K})\right)$. This implies that $C^{*}(X ; \mathbb{K})$ is a coproduct of shifts of $C^{*}(B ; \mathbb{K})$ and hence $C^{*}(X ; \mathbb{K})$ is in thick $_{D\left(C^{*}(B ; \mathbb{K})\right)}^{1}\left(C^{*}(B ; \mathbb{K})\right)$. On the other hand, for a spherical fibration $S^{l} \rightarrow X \rightarrow$ $B$, we obtain a characterization for the $C^{*}(B ; \mathbb{K})$-level of $C^{*}(X ; \mathbb{K})$ to be two; see Proposition 4.2. Combining the result with Proposition 2.5, we have the following proposition. 
Proposition 2.9. Let $B$ be a simply-connected space. Suppose that there exists a sequence of fibrations

$$
F_{1} \longrightarrow X_{1} \stackrel{p_{1}}{\longrightarrow} B, F_{2} \longrightarrow X_{2} \stackrel{p_{2}}{\longrightarrow} X_{1}, \ldots, F_{n} \longrightarrow X_{n} \stackrel{p_{n}}{\longrightarrow} X_{n-1},
$$

in which $X_{i}$ is simply-connected for $1 \leqslant i<n$ and $H^{*}\left(F_{i} ; \mathbb{K}\right) \cong H^{*}\left(S^{n_{i}} ; \mathbb{K}\right)$ for some $n_{i}$. Then one has inequalities

$$
\begin{gathered}
\operatorname{level}_{\mathrm{D}\left(C^{*}(B ; \mathbb{K})\right)}^{C^{*}(B ; \mathbb{K})} C^{*}\left(X_{n} ; \mathbb{K}\right) \leqslant 2^{n} \quad \text { and } \\
\operatorname{level}_{\mathrm{D}\left(C_{*}(\Omega B ; \mathbb{K})\right)}^{C_{*}(\Omega B ; \mathbb{K})} C_{*}\left(F_{f} ; \mathbb{K}\right) \leqslant 2^{n} \cdot \operatorname{dim} H_{*}(B ; \mathbb{K}),
\end{gathered}
$$

where $f=p_{n} \circ \cdots \circ p_{1}$.

In rational case, the result [32, Proposition 2.7] gives a better estimate of $C^{*}(B ; \mathbb{Q})$ level of $C^{*}\left(X_{n} ; \mathbb{Q}\right)$ than that of Proposition 2.9 provide each $n_{i}$ is odd.

In order to describe another main theorem, we recall a numerical invariant for DG modules related to the level. Let $A$ be a DG algebra. We call a morphism $f: M \rightarrow N$ in the derived category $\mathrm{D}(A)$ a ghost if $H(f)=0$. An object $M$ in $\mathrm{D}(A)$ is said to have ghost length $n$, denoted gh.len. $M=n$, if every composite

$$
M \stackrel{f_{1}}{\longrightarrow} Y_{1} \stackrel{f_{2}}{\longrightarrow} \cdots \stackrel{f_{n+1}}{\longrightarrow} Y_{n+1}
$$

of $n+1$ ghosts is trivial in $\mathrm{D}(A)$, and there exists a composite of $n$ ghosts from $M$ which is non-trivial in $\mathrm{D}(A)$; see $[\mathbf{2 2}]$.

The ghost length of a DG module $M$ gives a lower bound of the level of $M$.

Proposition 2.10 ([48, Lemma 6.7] [33, Proposition 7.5]). For any $M \in \mathrm{D}(A)$, one has

$$
\text { gh.len. } M+1 \leqslant \operatorname{level}_{\mathrm{D}(A)}^{A}(M) .
$$

Let $B G$ be the classifying space of a connected Lie group $G$. Since the diagonal map $\Delta: G \rightarrow G \times G$ is a homomorphism, it induces a map $B G \rightarrow B G^{\times 2}$, which is regarded as the diagonal map $B G \rightarrow B G \times B G$ under a homotopy equivalence between $B G^{\times 2}$ and $B G \times B G$. We give an estimate for the cochain type level of the composite

$$
\Delta^{(n-1)}: B G \stackrel{B \Delta}{\longrightarrow} B G^{\times 2} \longrightarrow \cdots \stackrel{B(1 \times \Delta)}{\longrightarrow} B G^{\times n}
$$

by considering the ghostness of a shriek map associated with the map $B(1 \times \Delta)$ : $B G^{\times l} \rightarrow B G^{\times(l+1)}$; see $[\mathbf{1 7}]$ and Section 5 for shriek maps on a Gorenstein space.

Theorem 2.11. Let $B G$ be the classifying space of a connected Lie group $G$ whose cohomology with coefficients in $\mathbb{K}$ is isomorphic to a polynomial algebra. Then in the derived category $\mathrm{D}\left(C^{*}\left(B G^{\times n}\right)\right)$, one has

$$
n-1 \leqslant \text { gh.len. } C^{*}(B G) .
$$

The assumption for a Lie group $G$ in Theorem 2.11 is satisfied for any field $\mathbb{K}$ if the homology $H_{*}(G ; \mathbb{Z})$ is torsion free. Moreover, the classical Lie groups $S O(n), \operatorname{Spin}(n)$ for $n \leqslant 9$, the exceptional Lie groups $G_{2}$ and $F_{4}$ satisfy the assumption in the case where the field $\mathbb{K}$ is of characteristic 2 while the integral homology groups of these Lie groups have 2-torsion; see [41]. 
The proof of Theorem 2.11 uses the Leray-Serre and the Eilenberg-Moore spectral sequences. The key to the proof is the non-triviality of the loop coproduct in string topology on the classifying space of a Lie group $[\mathbf{9}, \mathbf{3 4}]$. Therefore it is hard to expect an algebraic proof of the theorem.

By Proposition 2.10 and Theorem 2.11, we have the following result.

Proposition 2.12. Under the same assumption as in Theorem 2.11,

$$
n \leqslant \operatorname{level}_{\mathrm{D}\left(C^{*}\left(B G^{\times n}\right)\right.}^{C^{*}\left(B G^{\times n}\right)}\left(C^{*}(B G)\right) \leqslant(n-1) \operatorname{dim} Q H^{*}(B G ; \mathbb{K})+1,
$$

where $Q H^{*}(B G ; \mathbb{K})$ stands for the vector space of indecomposable elements of the algebra $H^{*}(B G ; \mathbb{K})$. Assume further that $Q H^{*}(B G ; \mathbb{K})^{2 j+1}=0$ for $j \geqslant 0$. Then

$$
\operatorname{level}_{\mathrm{D}\left(C^{*}\left(B G^{\times n}\right)\right)}^{C^{*}\left(B G^{\times n}\right)}\left(C^{*}(B G)\right)=(n-1) \operatorname{dim} Q H^{*}(B G ; \mathbb{K})+1 .
$$

For example, we consider the orthogonal group $S O(3)$. Since the mod 2 cohomology $H^{*}(B S O(3) ; \mathbb{Z} / 2)$ is a polynomial algebra generated by the second and the third Stiefel-Whitney classes, it follows that

$$
2 \leqslant \text { gh.len. } C^{*}(B S O(3) ; \mathbb{Z} / 2)+1 \leqslant \operatorname{level}_{\mathrm{D}\left(C^{*}\left(B S O(3)^{\times 2}\right)\right)}^{C^{*}\left(B S()^{\times 2}\right)}\left(C^{*}(B S O(3))\right) \leqslant 3 .
$$

Remark 2.13. Let $F_{\Delta^{(n-1)}}$ be the homotopy fibre of the map $\Delta^{(n-1)}: B G \rightarrow B G^{\times n}$. Then the fibration $F_{\Delta^{(n-1)}} \rightarrow B G$ admits the holonomy right action of $\Omega B G^{\times n}$ and is weakly equivalent to the fibration $B G \times{ }_{B G^{\times n}} E G^{\times n} \rightarrow B G$ with the holonomy right action of $G^{\times n}$; see, for example, [16, Proposition 2.11]. Then the duality in Theorem 2.4 (2) implies that

$$
\text { level }{ }_{\mathrm{D}\left(C^{*}\left(B G^{\times n}\right)\right)}^{C^{*}\left(B G^{\times n}\right)}\left(C^{*}(B G)\right)=\operatorname{level}_{\mathrm{D}\left(C_{*}\left(G^{\times n}\right)\right)}^{\mathbb{K}}\left(C_{*}\left(B G \times_{B G^{\times n}} E G^{\times n}\right)\right) .
$$

We observe that $B G \times{ }_{B G^{\times n}} E G^{\times n}$ is homotopy equivalent to a homogeneous space of the form $G^{\times n} / \Delta G=G^{\times(n-1)}$, where $\Delta: G \rightarrow G^{\times n}$ denotes the diagonal map. In fact, we have a homotopy fibre square

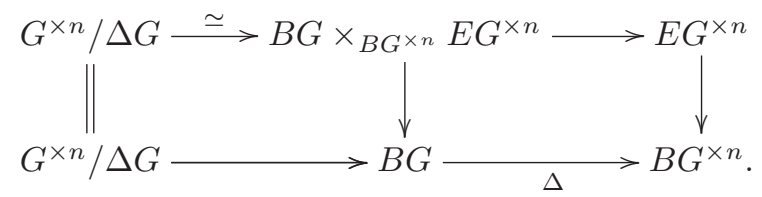

The rest of the article is organized as follows. In Section 3, we prove Theorem 2.4. Section 4 is devoted to proving Propositions 2.5, 2.8 and 2.9. Section 5 presents proofs of Theorem 2.11 and Proposition 2.12. In Appendix, we recall results on a coderived category due to Lefèvre-Hasegawa [38] on which we rely when proving Theorem 2.4. Moreover, a variant of Koszul duality due to He and Wu [21] is discussed.

\section{Proof of Theorem 2.4}

As we will see below, for an object $f$ in $\mathcal{T} O P_{B}$, we obtain quasi-isomorphisms which connect DG modules $C^{*}(s(f))$ and $C_{*}\left(F_{f}\right)$ by making use of the bar and cobar constructions. In order to prove Theorem 2.4, we incorporate such the quasiisomorphisms into arguments on appropriate derived and coderived categories. 
For a graded vector space $V$, we denote by $V^{\vee}$ the graded dual $\operatorname{Hom}_{\mathbb{K}}(V, \mathbb{K})$, namely $\left(V^{\vee}\right)^{-k}=\left(V^{\vee}\right)_{k}=\operatorname{Hom}_{\mathbb{K}}\left(V^{k}, \mathbb{K}\right)$. We say that $V$ is locally finite if $V^{i}$ is of finite dimension for each $i$.

Definition 3.1. (i) Let $A$ be an augmented DG algebra over $\mathbb{K}$ with differential of degree +1 and $\bmod -A$ the category of $\mathrm{DG}$ right $A$-modules. The derived category $\mathrm{D}(\bmod -A)$ of $\mathrm{DG}$ right $A$-modules is the localization of the homotopy category of $\bmod -A$ at the class of quasi-isomorphisms.

(ii) Let $C$ be a co-augmented DG coalgebra over $\mathbb{K}$ with differential of degree +1 and comod- $C$ the category of cocomplete DG right $C$-comodules; see the Appendix. The coderived category $\mathrm{D}$ (comod-C) of cocomplete $\mathrm{DG} C$-comodules is the localization of the homotopy category of comod $-C$ at the class of weak equivalences; see $[\mathbf{3 8}]$ and also the Appendix.

We shall write $\mathrm{D}(A)$ and $\mathrm{D}_{c}(C)$ for $\mathrm{D}(\bmod -A)$ and $\mathrm{D}($ comod $-C)$, respectively.

By definition, a simply-connected algebra $A$ satisfies the condition that $A^{0}=\mathbb{K}$, $A^{1}=0$ and $A^{i}=0$ for $i<0$. We call a coalgebra $C$ simply-connected if $C^{0}=\mathbb{K}$, $C^{-1}=0$ and $C^{i}=0$ for $i>0$. In what follows, we assume that an algebra and a coalgebra are endowed with an augmentation and a co-augmentation, respectively and that they are defined over a field $\mathbb{K}$.

Let $F$ : comod $-C \rightarrow C^{\vee}-$ mod be a functor given by sending a cocomplete DG right $C$-comodule to the DG left $C^{\vee}$-module with the same underlying $\mathbb{K}$-module and whose multiplication is given by the natural composite

$$
C^{\vee} \otimes M \rightarrow C^{\vee} \otimes M \otimes C \rightarrow C^{\vee} \otimes C \otimes M \rightarrow M .
$$

Composing the vector space dual functor ()$^{\vee}$ with $F$, we have an exact functor

$$
t D: \mathrm{D}(\operatorname{comod}-C) \stackrel{F_{*}}{\longrightarrow} \mathrm{D}\left(C^{\vee}-\bmod \right) \stackrel{()^{\vee}}{\longrightarrow} \mathrm{D}\left(\bmod -C^{\vee}\right)
$$

from the coderived category to the derived category; see Remark A.1.

We deal with the bar and cobar constructions below. For the (co)algebra and (co)module structures of these constructions, see [14, Section 2], [15, Section 4] and $[\mathbf{4 3}]$. We also refer the reader to $[\mathbf{2 3}]$ for differential graded objects.

Let $A$ be a DG algebra and consider the bar resolution $B(A ; A) \rightarrow{ }_{A} \mathbb{K}$ of $\mathbb{K}$. Let $B(A)$ be a DG coalgebra defined by $B(A)=\mathbb{K} \otimes_{A} B(A ; A)$. By using the twisted tensor product construction associated with the natural twisting cochain $\tau: B(A) \rightarrow$ $A$ of degree +1 , we have a pair of adjoint functors

$$
\mathrm{D}_{c}(B(A)) \underset{R:=-\otimes_{\tau} B(A)}{\stackrel{L:=-\otimes_{\tau} A}{<}} \mathrm{D}(A) .
$$

For more details, see $[\mathbf{4 3}],\left[\mathbf{3 8}\right.$, Ch. 2], $[\mathbf{2 8}]$ and also Appendix. We write $R_{A}$ for the functor $-\otimes_{\tau} B(A)$. The definition of the twisted tensor product enables us to deduce that $R_{A}$ coincides with the functor $-\otimes_{A} B(A ; A)$. For a right $A$-module $M$, we may write $B(M ; A)$ for $M \otimes_{A} B(A ; A)$. For a coalgebra $C$ and a right $C$-comodule $N$, let $\Omega(N ; C)$ denote the cobar construction; see, for example, [14, Section 2].

The duality on the bar and cobar constructions yields the following result. 
Proposition 3.2. Let $C$ be a simply-connected $D G$ coalgebra with $H(C)$ locally finite. Then there exists an equivalence

$$
\Theta: \mathrm{D}(\Omega C) \rightarrow \mathrm{D}\left(B\left(C^{\vee}\right)^{\vee}\right)
$$

of triangulated categories such that for a $D G C$-comodule $N$ with $H(N)$ locally finite and bounded above,

$$
\Theta(\Omega(N ; C)) \cong B\left(N^{\vee} ; C^{\vee}\right)^{\vee}
$$

Proof. Let $u: A=T V \stackrel{\simeq}{\longrightarrow} C^{\vee}$ be a TV-model for the simply-connected DG algebra $C^{\vee}$ in the sense of Halperin and Lemaire [20]. By assumption, $H\left(C^{\vee}\right)$ is locally finite. Then without loss of generality, we can assume that $A$ is also locally finite; see $[\mathbf{1 4}$, Proposition 4.2].

Let $\Delta: C \rightarrow C \otimes C$ be the comultiplication on $C$. Then the multiplication $m: C^{\vee} \otimes$ $C^{\vee} \rightarrow C^{\vee}$ is defined by the composite

$$
C^{\vee} \otimes C^{\vee}=C^{\vee} \otimes C^{\vee} \stackrel{q^{\prime}}{\rightarrow}(C \otimes C)^{\vee} \stackrel{\Delta}{\rightarrow} C^{\vee}=C^{\vee},
$$

where $q^{\prime}$ denotes the natural quasi-isomorphism. We have a commutative diagram

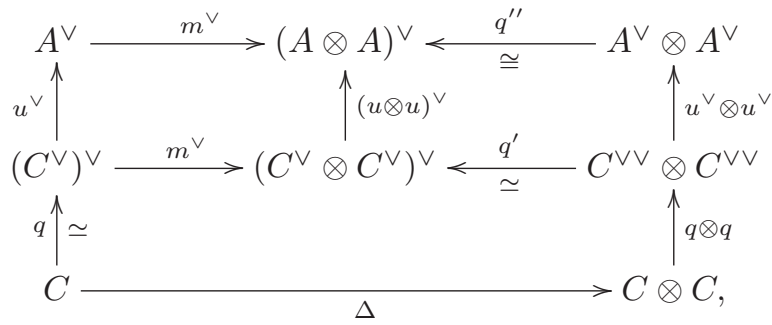

where $q$ and $q^{\prime \prime}$ are the natural quasi-isomorphisms. In fact, the commutativity of the lower square follows from that of the diagram

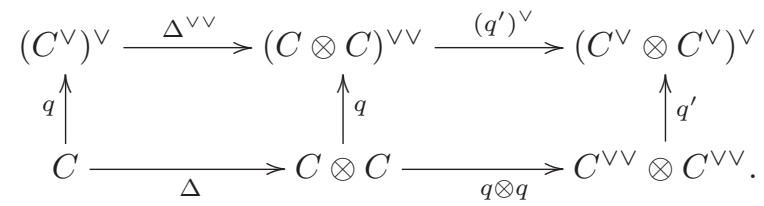

Observe that $q^{\prime \prime}: A^{\vee} \otimes A^{\vee} \rightarrow(A \otimes A)^{\vee}$ is an isomorphism because $A$ is locally finite. This implies that $u^{\vee} \circ q: C \rightarrow A^{\vee}$ is a quasi-isomorphism of coalgebras. We then have a sequence of quasi-isomorphisms of algebras

$$
\Omega C \stackrel{\rho:=\Omega\left(u^{\vee} q\right)}{\simeq} \Omega\left(A^{\vee}\right) \underset{\simeq}{\stackrel{\mu_{1}}{\simeq}} \rightarrow B(A)^{\vee} \underset{\simeq}{\simeq} B\left(C^{\vee}\right)^{\vee} .
$$

Thus the result [30, Proposition 4.2] enables us to obtain equivalences of triangulated categories

$$
\mathrm{D}(\Omega C) \underset{\rho^{*}}{\stackrel{-\otimes_{\Omega C}^{L} \Omega\left(A^{\vee}\right)}{\longrightarrow}} \stackrel{-\otimes_{\Omega\left(A^{\vee}\right)}^{L} B(A)^{\vee}}{\underset{\simeq}{\simeq}} \mathrm{D}\left(\Omega\left(A^{\vee}\right)\right) \underset{\mu_{1}^{*}}{\stackrel{\sim}{\longleftarrow}} \mathrm{D}\left(B(A)^{\vee}\right) \underset{\mu_{2}^{*}}{\simeq} \mathrm{D}\left(B\left(C^{\vee}\right)^{\vee}\right) .
$$

We define $\Theta: \mathrm{D}(\Omega C) \rightarrow \mathrm{D}\left(B\left(C^{\vee}\right)^{\vee}\right)$ by the composite. 
Let $C_{1} \stackrel{\simeq}{\longrightarrow} N^{\vee}$ be an $A$-semifree resolution for $N^{\vee}$; see [14, Propositions 4.6 and 4.7]. Since $H_{*}(N)$ is locally finite, we may assume that so is the $A$-module $C_{1}$; see $[\mathbf{1 4}$, Proposition 4.6]. Then we can define a comodule structure on $C_{1}^{\vee}$ by the composite $c: C_{1}^{\vee} \longrightarrow\left(C_{1} \otimes A\right)^{\vee} \cong C_{1}^{\vee} \otimes A^{\vee}$. The same argument as above allows us to obtain a commutative diagram

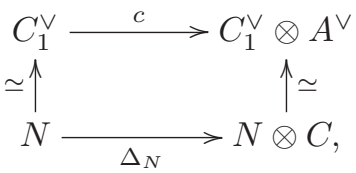

in which vertical arrows are quasi-isomorphisms. Thus we have an isomorphism $\Omega(N ; C) \cong \rho^{*} \Omega\left(C_{1}^{\vee} ; A^{\vee}\right)$ in $\mathrm{D}(\Omega C)$. Moreover, it follows from the locally finiteness of $C_{1}$ and $A$ that $\Omega\left(C_{1}^{\vee} ; A^{\vee}\right)$ is isomorphic to $\mu_{1}^{*}\left(B\left(C_{1} ; A\right)^{\vee}\right)$ and $\mu_{2}^{*}\left(B\left(C_{1} ; A\right)^{\vee}\right) \cong$ $B\left(N^{\vee} ; C^{\vee}\right)^{\vee}$ in $\mathrm{D}\left(B\left(C^{\vee}\right)^{\vee}\right)$. This completes the proof.

We have a crucial result on exact functors which connect the triangulated categories $\mathrm{D}(\Omega C)$ and $\mathrm{D}\left(C^{\vee}\right)$ for a coalgebra $C$. The result is a key to proving the duality on chain and cochain type levels described in Theorem 2.4.

Let $A$ and $C$ be an augmented DG algebra and a co-augmented cocomplete DG coalgebra, respectively. The result [14, Proposition 2.14] asserts that for a $C$ comodule $N$, there exist a quasi-isomorphism $\sigma_{C}: C \stackrel{\widetilde{\Im}}{\rightarrow} B \Omega C$ of coalgebras and a quasi-isomorphism $\sigma_{N}: N \stackrel{\widetilde{\sim}}{\rightarrow} B(\Omega(N ; C) ; \Omega C)$ of $C$-comodules.

Theorem 3.3. (i) Under the same assumption as above on the coalgebra $C$, one has a commutative diagrams up to isomorphism

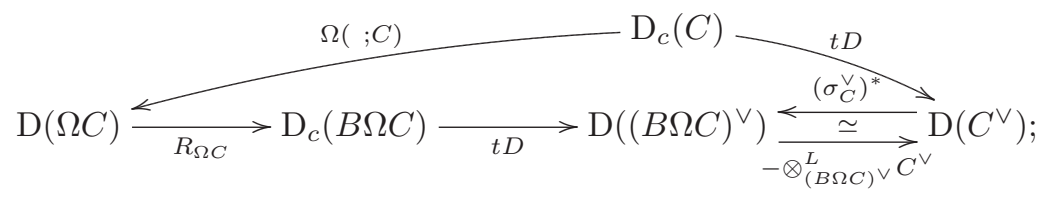

that is, there exists a natural isomorphism between two composite functors from $\mathrm{D}_{c}(C)$ to $\mathrm{D}\left((B \Omega C)^{\vee}\right)$. Moreover, all the functors between (co)derived categories are exact.

(ii) Let $C$ be a simply-connected $D G$ coalgebra with $H(C)$ locally finite. Let $\mathrm{D}_{c}^{l f,-}(C)$ denote the full subcategory of $\mathrm{D}_{c}(C)$ consisting of comodules whose cohomologies are locally finite and bounded above. Then one has a commutative diagram up to isomorphism

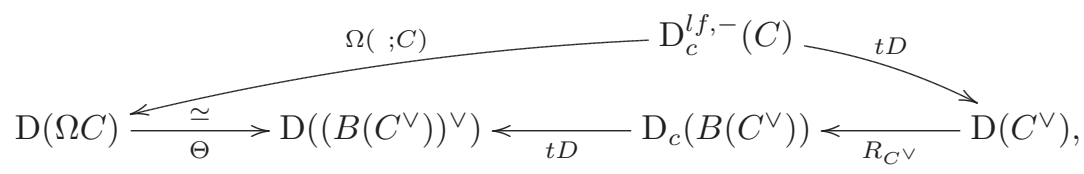

in which all the functors are exact.

Proof. (i) Let $\tau: C \rightarrow \Omega C$ be the canonical twisting cochain. Then $\Omega(; C)$ is nothing but the functor $L=-\otimes_{\tau} \Omega C$ mentioned in Theorem A.2 below. In particular, we see 
that $\Omega(; C)$ is exact. Moreover, it follows that for any $N$ in $\mathrm{D}_{c}(C)$,

$$
t D(B(\Omega(N ; C) ; \Omega C))=B(\Omega(N ; C) ; \Omega C)^{\vee} \simeq N^{\vee}=\left(\sigma_{C}^{\vee}\right)^{*} t D(N)
$$

in $\mathrm{D}\left((B \Omega C)^{\vee}\right)$. This implies that the diagram is commutative up to isomorphism.

(ii) Proposition 3.2 yields that for any $N$ in $\mathrm{D}_{c}^{l f,-}(C)$,

$$
\Theta(\Omega(N ; C)) \cong B\left(N^{\vee} ; C^{\vee}\right)^{\vee}=\left(t D \circ R_{C^{\vee}} \circ t D\right)(N) .
$$

We have the result.

In order to prove Theorem 2.4, we recall important results on the level.

Lemma 3.4 ([48, Lemma 3.9]). Let $M$ be a DG-module over a non-negative simplyconnected or non-positive connected DG algebra A. Assume that $M$ is bounded below if $A$ is non-negative and is bounded above if $A$ is non-positive. Then

$$
\operatorname{dim} H\left(M \otimes_{A}^{L} \mathbb{K}\right) \geqslant \operatorname{level}_{\mathrm{D}(A)}^{A}(M) .
$$

The difference between the dimension of $H\left(M \otimes_{A}^{L} \mathbb{K}\right)$ and the level is also of interest to us. In general, the difference is very large. The proof of Lemma 3.4 which we provide below exhibits the fact.

Proof of Lemma 3.4. If $\operatorname{dim} H\left(M \otimes_{A}^{L} \mathbb{K}\right)=\infty$, then the assertion is immediate.

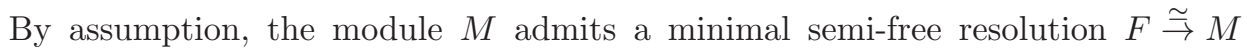
endowed with a filtration $\left\{F^{l}\right\}_{l \geqslant 0}$ of $F$; see $[\mathbf{1 3}, \mathbf{1 6}]$ and $[\mathbf{1 5}$, Section 2]. We thus obtain triangles $\coprod_{i} \Sigma^{n_{i}^{0}} A \rightarrow F^{1} \rightarrow \coprod_{j} \Sigma^{n_{j}^{1}} A \rightarrow, F^{1} \rightarrow F^{2} \rightarrow \coprod_{j} \Sigma^{n_{j}^{2}} A \rightarrow, \ldots, F^{n-1} \rightarrow$ $F^{n} \rightarrow \coprod_{j} \Sigma^{n_{j}^{n}} A \rightarrow, \ldots$ The minimality of the semi-free resolution enables us to deduce that

$$
H\left(M \otimes_{A}^{L} \mathbb{K}\right)=H\left(F \otimes_{A} \mathbb{K}\right)=F \otimes_{A} \mathbb{K}=\coprod_{s \geqslant 0} \coprod_{j} \Sigma^{n_{j}^{s}} \mathbb{K}
$$

Suppose that $\operatorname{dim} H\left(M \otimes_{A}^{L} \mathbb{K}\right)$ is of finite dimension. Then it follows that there exists an integer $n$ such that $F^{n} \simeq M$ and each index $j$ runs in finite numbers. Thus we see that $M \in \operatorname{thick}_{\mathrm{D}(A)}^{n+1}(A)$. If $n_{j}^{s}=0$ for any $j$ and $s$, then $M \simeq 0$ and hence the result is obvious. Without loss of generality, we can assume that for any $s, \coprod_{j} \Sigma^{n_{j}^{s}} A$ is non-trivial. We then have

$$
\operatorname{dim} H\left(M \otimes{ }_{A}^{L} \mathbb{K}\right)=\operatorname{dim} \coprod_{s=0}^{n} \coprod_{j} \Sigma^{n_{j}^{s}} \mathbb{K} \geqslant n+1 \geqslant \operatorname{level}_{\mathrm{D}(A)}^{A}(M) .
$$

This completes the proof.

Let $\gamma: \mathcal{T} \rightarrow \mathcal{U}$ be an exact functor of triangulated categories. Then we have the following result.

Lemma 3.5 ([2, Theorem 2.4(6)]). $\operatorname{level}_{\mathcal{T}}^{C}(M) \geqslant \operatorname{level}_{\mathcal{U}}^{\gamma(C)}(\gamma(M))$. 
Let $B$ be a simply-connected space and $f: X \rightarrow B$ a map. Recall from $[\mathbf{1 4}$, Theorem II] a quasi-isomorphism of DG-modules

$$
\Phi: \Omega\left(C_{*}(X) ; C_{*}(B)\right) \stackrel{\simeq}{\longrightarrow} C_{*}\left(F_{f}\right),
$$

which is compatible with actions of $\Omega C_{*}(B)$ and $C_{*}(\Omega B)$ via a quasi-isomorphism of DG algebras

$$
\phi: \Omega C_{*}(B) \rightarrow C_{*}(\Omega B),
$$

where $\Omega\left(N ; C_{*}(B)\right)$ denotes the cobar construction of the right $C_{*}(B)$-comodule $N$. We mention that the quasi-isomorphisms $\Phi$ and $\phi$ are induced from the universal constructions due to Adams [1]; see also [14, Section 3].

We connect the category $\mathcal{T} \mathcal{O} \mathcal{P}_{B}$ with $\mathrm{D}\left(C_{*}(\Omega B)\right)$ and $\mathrm{D}\left(\Omega C_{*}(B)\right)$.

Proposition 3.6. Let $B$ be a simply-connected space. One has a commutative diagram up to isomorphism

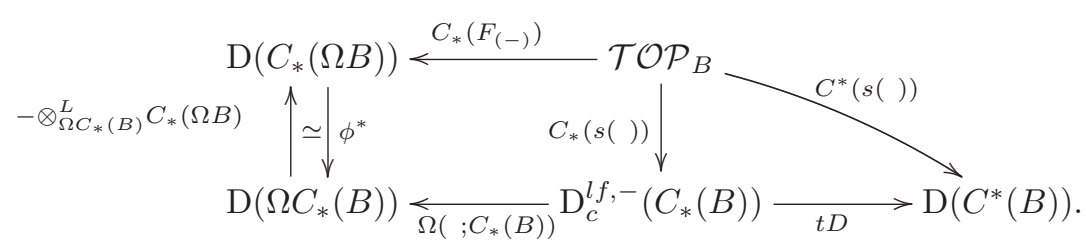

Proof. The quasi-isomorphisms in (3.1) and (3.2) enable us to conclude that the left hand-side square is commutative up to isomorphism. By definition, the right handside triangle is commutative.

Remark 3.7. We write $\eta$ and $\nu$ for the composites $-\otimes_{(B \Omega C)^{\vee}}^{L} C^{\vee} \circ t D \circ R_{\Omega C_{*}(B)} \circ \phi^{*}$ and $-\otimes_{\Omega C_{*}(B)}^{L} C_{*}(\Omega B) \circ \Theta^{-1} \circ t D \circ R_{C^{*}(B)}$, respectively; see Theorem 3.3 and Proposition 3.6. Then it follows that $\eta\left(C_{*}(\Omega B)\right) \cong \eta\left(C_{*}\left(F_{(* \rightarrow B)}\right)\right) \cong C^{*}(*) \cong \mathbb{K}_{C^{*}(B)}$ and

$$
\nu\left(C^{*}(B)\right) \cong \nu C^{*}(s(i d: B \rightarrow B)) \cong C_{*}\left(F_{(i d)}\right) \cong C_{*}(*) \cong \mathbb{K}_{C_{*}(\Omega B)} .
$$

Moreover, we see that $\eta(\mathbb{K}) \cong \eta\left(C_{*}\left(F_{(i d: B \rightarrow B)}\right)\right) \cong C^{*}(B)$ and

$$
\nu(\mathbb{K}) \cong \nu\left(C^{*}(s(* \rightarrow B))\right) \cong C_{*}\left(F_{(* \rightarrow B)}\right) \cong C_{*}(\Omega B) .
$$

We are now ready to prove our main theorem.

Proof of Theorem 2.4. It follows from [16, Proposition 19.2] that $B\left(C_{*}(\Omega B) ; C_{*}(\Omega B)\right)$ is a $C_{*}(\Omega B)$-semifree resolution of $\mathbb{K}$. Then the result $[\mathbf{1 5}$, Proposition 6.7$]$ yields that

$$
C_{*}(X) \simeq C_{*}\left(F_{f}\right) \otimes_{C_{*}(\Omega B)} B\left(C_{*}(\Omega B) ; C_{*}(\Omega B)\right) \simeq C_{*}\left(F_{f}\right) \otimes_{C_{*}(\Omega B)}^{L} \mathbb{K} .
$$

By virtue of Lemma 3.4, we have the first inequality.

Let $F_{f} \rightarrow E_{X} \rightarrow B$ be the fibration associated with the map $f: X \rightarrow B$. Since there exists a homotopy equivalence $j: X \rightarrow E_{X}$ which is in $\mathcal{T} O P_{B}$, it follows that, as vector spaces,

$$
H^{*}\left(C^{*}(X) \otimes_{C^{*}(B)}^{L} \mathbb{K}\right) \cong \operatorname{Tor}_{C^{*}(B)}\left(C^{*}(X), \mathbb{K}\right) \cong \operatorname{Tor}_{C^{*}(B)}\left(C^{*}\left(E_{X}\right), \mathbb{K}\right) \cong H^{*}\left(F_{f}\right) .
$$

Observe that the third isomorphism is induced by the Eilenberg-Moore map; see, for example, [19, Theorem 3.3]. By applying Lemma 3.4 again, one has the second inequality. 
It follows from Lemma 3.5, Theorem 3.3 (i), Proposition 3.6 and Remark 3.7 that

$$
\operatorname{level}_{\mathrm{D}\left(C_{*}(\Omega B)\right)}^{C_{*}(\Omega B)}\left(C_{*}\left(F_{f}\right)\right) \geqslant \operatorname{level}_{\mathrm{D}\left(C^{*}(B)\right)}^{\mathbb{K}}\left(C^{*}(X)\right) .
$$

Theorem 3.3 (ii) yields the converse inequality. The same argument as above works well to obtain the equality in (2).

Remark 3.8. Let $C$ be a co-augmented cocomplete $D G$ coalgebra with $H(C)$ locally finite and $M$ an object in $\mathrm{D}^{l f,-}(C)$. Then Theorem 3.3 yields algebraic versions of equalities in Theorem 2.4. Indeed, we have equalities

$$
\begin{gathered}
\text { level }_{\mathrm{D}(\Omega C)}^{\Omega C}(\Omega(M ; C))=\text { level }_{\mathrm{D}\left(C^{\vee}\right)}^{\mathbb{K}}\left(M^{\vee}\right) \text { and } \\
\text { level }_{\mathrm{D}\left(C^{\vee}\right)}^{C^{\vee}}\left(M^{\vee}\right)=\text { level }_{\mathrm{D}(\Omega C)}^{\mathbb{K}}(\Omega(M ; C)) .
\end{gathered}
$$

Remark 3.9. As mentioned in the Introduction, the string topology category $\mathrm{St}_{M}$ for a simply-connected oriented manifold $M$ is a full subcategory of $\mathrm{D}\left(C_{*}(\Omega M)\right)$; see [49]. Then Proposition 3.6 and Theorem 3.3 may generalize the result [6, Theorem 2.8] on the Dwyer-Kan equivalence between $\mathrm{St}_{M}$ and the full subcategory of $\mathrm{D}\left(C^{*}(M)\right)$ modules consisting of objects in the image of the functor $C^{*}(s())$. This will be discussed in a forthcoming paper [35].

\section{Proofs of Propositions 2.5, 2.8 and 2.9}

We here recall some full subcategories of a triangulated category $\mathcal{T}$ before proving Proposition 2.5.

Let $\mathcal{A}$ be a subcategory of $\mathcal{T}$ and $\operatorname{add}^{\Sigma}(\mathcal{A})$ the smallest full subcategory of $\mathcal{T}$ that contains $\mathcal{A}$ and is closed under finite coproducts, all shifts and isomorphisms. The category $\operatorname{smd}(\mathcal{A})$ is defined to be the smallest full subcategory of $\mathcal{T}$ that contains $\mathcal{A}$ and is closed under retracts. For full subcategories $\mathcal{A}$ and $\mathcal{B}$ of $\mathcal{T}$, let $\mathcal{A} * \mathcal{B}$ be the full subcategory whose objects $L$ occur in a triangle $M \rightarrow L \rightarrow N \rightarrow \Sigma M$ with $M \in \mathcal{A}$ and $N \in \mathcal{B}$. Then we see that $\operatorname{thick}_{\mathcal{T}}^{n}(C)=\operatorname{smd}\left(\operatorname{add}^{\Sigma}(C)^{* n}\right)$; see [7] and $[\mathbf{2}, 2.2 .1]$.

A triangular inequality on levels is described in the following lemma.

Lemma 4.1 (cf. [48, The proof of 6.3.2(3)]). Let $\mathcal{T}$ be a triangulated category and $C, C^{\prime}$ objects in $\mathcal{T}$. If level $\mathrm{T}_{\mathcal{T}}^{C} M \leqslant n$ and level $\mathrm{T}_{\mathcal{T}}^{C^{\prime}} C \leqslant l$, then level ${ }_{\mathcal{T}}^{C^{\prime}} M \leqslant n l$.

Proof. It suffices to prove that if $M \in \operatorname{thick}_{\mathcal{T}}^{n}(C)$ and $C \in \operatorname{thick}_{\mathcal{T}}^{l}\left(C^{\prime}\right)$, then $M \in$ $\operatorname{thick}_{\mathcal{T}}^{n l}\left(C^{\prime}\right)$.

Since the thickening thick ${ }_{\mathcal{T}}^{l}\left(C^{\prime}\right)$ is closed under finite coproducts, all shifts and retracts, it follows that $\operatorname{add}^{\Sigma}(C) \subset \operatorname{thick}_{\mathcal{T}}^{l}\left(C^{\prime}\right)$ and hence $\operatorname{thick}_{\mathcal{T}}^{1}(C) \subset \operatorname{thick}_{\mathcal{T}}^{l}\left(C^{\prime}\right)$. Assume that $\operatorname{thick}_{\mathcal{T}}^{i}(C) \subset \operatorname{thick}_{\mathcal{T}}^{i l}\left(C^{\prime}\right)$ for $i \leqslant n-1$. For any object $M \in \operatorname{thick}_{\mathcal{T}}^{n}(C)$, there exists a triangle $M_{1} \rightarrow M^{\prime} \rightarrow M_{2} \rightarrow \Sigma M_{1}$ such that $M$ is a retract of $M^{\prime}$, $M_{1} \in \operatorname{thick}_{\mathcal{T}}^{n-1}(C)$ and $M_{2} \in \operatorname{thick}_{\mathcal{T}}^{1}(C)$. This yields that

$$
\begin{aligned}
M & \in \operatorname{smd}\left(\operatorname{thick}_{\mathcal{T}}^{(n-1)}(C) * \operatorname{thick}_{\mathcal{T}}^{1}(C)\right) \\
& \subset \operatorname{smd}\left(\operatorname{smd}\left(\operatorname{add}^{\Sigma}\left(C^{\prime}\right)^{*(n-1) l}\right) * \operatorname{smd}\left(\operatorname{add}^{\Sigma}\left(C^{\prime}\right)^{* l}\right)\right) \\
& =\operatorname{smd}\left(\operatorname{add}^{\Sigma}\left(C^{\prime}\right)^{*(n-1) l} * \operatorname{add}^{\Sigma}\left(C^{\prime}\right)^{* l}\right) \\
& =\operatorname{thick}_{\mathcal{T}}^{n l}\left(C^{\prime}\right) .
\end{aligned}
$$

Observe that the first equality follows from [7, Lemma 2.2.1]. This completes the 
proof.

Proof of Proposition 2.5. Lemma 4.1 and Theorem 2.4 induce the inequalities. In fact, we see that

$$
\begin{aligned}
\operatorname{level}_{\mathrm{D}\left(C_{*}(\Omega B)\right)}^{C_{*}(\Omega B)}\left(C_{*}\left(F_{f}\right)\right) & =\operatorname{level}_{\mathrm{D}\left(C^{*}(B)\right)}^{\mathbb{K}}\left(C^{*}(X)\right) \\
& \leqslant \operatorname{level}_{\mathrm{D}\left(C^{*}(B)\right)}^{\mathbb{K}}\left(C^{*}(B)\right) \cdot \operatorname{level}_{\mathrm{D}\left(C^{*}(B)\right)}^{C^{*}(B)}\left(C^{*}(X)\right) \\
& =\operatorname{level}_{\mathrm{D}\left(C_{*}(\Omega B)\right)}^{C_{*}(\Omega B)}\left(\mathbb{K}^{\mathbb{K}}\right) \cdot \operatorname{level}_{\mathrm{D}\left(C^{*}(B)\right)}^{C^{*}(B)}\left(C^{*}(X)\right) \\
& \leqslant \operatorname{dim} H_{*}(B) \cdot \operatorname{level}_{\mathrm{D}\left(C^{*}(B)\right)}^{C^{*}(B)}\left(C^{*}(X)\right)
\end{aligned}
$$

We here observe that $C_{*}\left(F_{i d}\right) \cong \mathbb{K}$ in $\mathrm{D}\left(C_{*}(\Omega B)\right)$ for the homotopy fibre $F_{i d}$ of the identity map on $B$. The second inequalities follow from the same consideration as above. Observe that the based loop space $\Omega B$ is the homotopy fibre of the map $* \rightarrow B$.

Proof of Proposition 2.8. By replacing the square

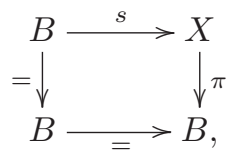

which is homotopy commutative, to a totally fibred square, we have a commutative diagram

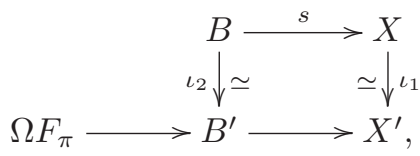

in which $\iota_{1}$ and $\iota_{2}$ are homotopy equivalences and bottom sequence is a fibration; see [45, Propositions 3.2.2 and 3.2.3]. The map $\iota_{1}$ gives rise to an equivalence

$$
C^{*}\left(\iota_{1}\right)^{*}: \mathrm{D}\left(C^{*}(X)\right) \rightarrow \mathrm{D}\left(C^{*}\left(X^{\prime}\right)\right)
$$

of triangulated categories. It is readily seen that $C^{*}\left(\iota_{1}\right)^{*}\left(C^{*}(B)\right) \cong C^{*}\left(B^{\prime}\right)$. This yields that

$$
\text { level }_{\mathrm{D}\left(C^{*}\left(X^{\prime}\right)\right)}^{C^{*}\left(X^{\prime}\right)} C^{*}\left(B^{\prime}\right)=\operatorname{level}_{\mathrm{D}\left(C^{*}(X)\right)}^{C^{*}(X)} C^{*}(B) .
$$

In view of the Leray-Serre spectral sequence of the path-loop fibration $\Omega F_{\pi} \rightarrow$ $P F_{\pi} \rightarrow F_{\pi}$, we see that $H^{*}\left(F_{\pi} ; \mathbb{K}\right)=\mathbb{K}$ if and only if $H^{*}\left(\Omega F_{\pi} ; \mathbb{K}\right)$ and $H^{*}\left(F_{\pi} ; \mathbb{K}\right)$ are of finite dimension. Observe that $F_{\pi}$ is simply-connected since $\pi$ has a right inverse. By Corollary 2.6 (2), we have the result.

Before proving Proposition 2.9, we consider a special case for the assertion.

Proposition 4.2. Let $F \rightarrow X \rightarrow B$ be a fibration with $B$ simply-connected. Suppose that $H^{*}(F ; \mathbb{K}) \cong H^{*}\left(S^{l} ; \mathbb{K}\right)$ as a graded vector space. Then one has

$$
\text { level }_{\mathrm{D}\left(C^{*}(B ; \mathbb{K})\right)}^{C_{*}(B ; \mathbb{K})} C^{*}(X ; \mathbb{K}) \leqslant 2 .
$$

Moreover, level ${ }_{\mathrm{D}\left(C^{*}(B ; \mathbb{K})\right)}^{C^{*}(B ; \mathbb{K})} C^{*}(X ; \mathbb{K})=2$ if and only if $H^{*}(X ; \mathbb{K})$ is not a free $H^{*}(B ; \mathbb{K})$ module. 
The following lemma serves to prove Proposition 4.2.

Lemma 4.3. Let $X \rightarrow B$ be an object in $\mathcal{T} O P_{B}$. Then level ${ }_{\mathrm{D}\left(C^{*}(B)\right)}^{C_{*}(B)} C^{*}(X)=1$ if and only if $H^{*}(X ; \mathbb{K})$ is a free $H^{*}(B ; \mathbb{K})$-module.

Proof. Suppose that level ${ }_{\mathrm{D}\left(C^{*}(B)\right)}^{C_{*}(B)} C^{*}(X)=1$. Then by definition, we see that $C^{*}(X)$ is a retract of a free $C^{*}(B)$-module. Therefore $H^{*}(X)$ is a projective $H^{*}(B)$-module and hence $H^{*}(X)$ is a free $H^{*}(B)$-module; see, for example, [16, page 274, Remark 1].

We see that level ${ }_{\mathrm{D}\left(H^{*}(B)\right)}^{H_{*}(B)} H^{*}(X)=1$ if $H^{*}(X)$ is a free $H^{*}(B)$-module. The result $\left[33\right.$, Corollary 7.3] implies that $\operatorname{level}_{\mathrm{D}\left(C^{*}(B)\right)}^{C_{*}(B)} C^{*}(X)$ is less than or equal to the level level $_{\mathrm{D}\left(H^{*}(B)\right)}^{H_{*}(B)} H^{*}(X)$. This completes the proof.

Proof of Proposition 4.2. Let $S_{l}$ be the homotopy fibre of the projection $X \rightarrow B$. We observe that $H^{*}\left(S_{l} ; \mathbb{K}\right) \cong H^{*}(F ; \mathbb{K}) \cong H^{*}\left(S^{l} ; \mathbb{K}\right)$. In order to prove the proposition, it suffices to show that level $\frac{\mathbb{K}\left(C_{*}(\Omega B)\right)}{\mathbb{K}} C_{*}\left(S_{l}\right) \leqslant 2$. This follows from Theorem $2.4(2)$. We define a DG subalgebra $R$ of $C_{*}(\Omega B)$ by $R_{0}=\mathbb{K}, R_{1}=$ Ker $d$ and $R_{\geqslant 2}=C_{\geqslant 2}(\Omega B)$. It is immediate that the inclusion $i: R \rightarrow C_{*}(\Omega B)$ is a quasi-isomorphism. Then the map $i$ induces an equivalence of categories $i^{*}: \mathrm{D}\left(C_{*}(\Omega B)\right) \rightarrow \mathrm{D}(R)$. Moreover, we have $i^{*}(\mathbb{K})=\mathbb{K}$ and $i^{*}\left(C_{*}\left(S_{l}\right)\right)=C_{*}\left(S_{l}\right)$. Therefore, we conclude that

$$
\operatorname{level}_{\mathrm{D}\left(C_{*}(\Omega B)\right)}^{\mathbb{K}} C_{*}\left(S_{l}\right)=\operatorname{level}_{\mathrm{D}\left(C_{*}(R)\right)}^{\mathbb{K}} C_{*}\left(S_{l}\right) .
$$

Let $N$ be a DG $R$-submodule of $C_{*}\left(S_{l}\right)$ defined by $N_{\leqslant l-1}=0, N_{l}=\operatorname{Im} d$ and $N_{i}=$ $C_{i}\left(S_{l}\right)$ for $i>l$. Since $N$ is acyclic, it follows that the projection $C_{*}\left(S_{l}\right) \rightarrow C_{*}\left(S_{l}\right) / N$ is a quasi-isomorphism of $R$-modules. Moreover, we can construct a triangle in $\mathrm{D}(R)$ of the form $\Sigma^{-l} \mathbb{K} \rightarrow C_{*}\left(S_{l}\right) / N \rightarrow \mathbb{K} \rightarrow$. In fact, the projection from the quotient $\left(C_{*}\left(S_{l}\right) / N\right) / \Sigma^{-l} \mathbb{K}$ to $\mathbb{K}$ is a quasi-isomorphism of $R$-modules. Then the triangle yields that level $\underset{\mathrm{D}(R)}{\mathbb{K}} C_{*}\left(S_{l}\right)=$ level $_{\mathrm{D}(R)}^{\mathbb{K}} C_{*}\left(S_{l}\right) / N \leqslant 2$. We have the result. The latter half of the assertion follows from Lemma 4.3 .

Remark 4.4. We can prove Proposition 4.2 by means of a minimal semifree resolution $\Gamma \stackrel{\sim}{\rightarrow} C^{*}(X)$ of $C^{*}(X)$ as a $C^{*}(B)$-module. Indeed, we see that

$$
H^{*}\left(\mathbb{K} \otimes_{C^{*}(B)} \Gamma\right)=H^{*}\left(\mathbb{K} \otimes_{C^{*}(B)}^{L} C^{*}(X)\right)=H^{*}\left(S^{l}\right)=\mathbb{K}\{1, w\},
$$

where $\operatorname{deg} 1=0$ and $\operatorname{deg} w=l$. This implies that the filtration of $F$ has class at most 2 ; see $[\mathbf{2}, 4.1]$. Proposition 4.2 follows from [2, Theorem 4.1].

We use again Lemma 4.1 to prove Proposition 2.9 .

Proof of Proposition 2.9. Consider the maps $C^{*}(B) \stackrel{\alpha}{\rightarrow} C^{*}\left(X_{i}\right) \stackrel{p_{i+1}^{*}}{\rightarrow} C^{*}\left(X_{i+1}\right)$, where $\alpha=\left(p_{i} \circ \cdots \circ p_{1}\right)^{*}$. Then the map $\alpha$ induces an exact functor $\alpha^{*}: \mathrm{D}\left(C^{*}\left(X_{i}\right)\right) \rightarrow$ $\mathrm{D}\left(C^{*}(B)\right)$. In view of Proposition 4.2 and Lemma 3.5, we have

$$
2 \geqslant \operatorname{level}_{\mathrm{D}\left(C^{*}\left(X_{i}\right)\right)}^{C^{*}\left(X_{i}\right)} C^{*}\left(X_{i+1}\right) \geqslant \operatorname{level}_{\mathrm{D}\left(C^{*}(B)\right)}^{\alpha^{*} C^{*}\left(X_{i}\right)} \alpha^{*} C^{*}\left(X_{i+1}\right)=\operatorname{level}_{\mathrm{D}\left(C^{*}(B)\right)}^{\alpha^{*} C^{*}\left(X_{i}\right)} C^{*}\left(X_{i+1}\right) .
$$

Therefore, Lemma 4.1 and the induction hypothesis allow us to deduce that

$$
\text { level }_{\mathrm{D}\left(C^{*}(B)\right)}^{C^{*}(B)} C^{*}\left(X_{i+1}\right) \leqslant \operatorname{level}_{\mathrm{D}\left(C^{*}(B)\right)}^{C^{*}(B)} \alpha^{*} C^{*}\left(X_{i}\right) \cdot \text { level }_{\mathrm{D}\left(C^{*}(B)\right)}^{\alpha^{*} C^{*}\left(X_{i}\right)} C^{*}\left(X_{i+1}\right) \leqslant 2^{i} \cdot 2 .
$$

We have the first inequality. The second inequality follows from Proposition 2.5. 


\section{Proofs of Theorem 2.11 and Proposition 2.12}

We begin by recalling a shriek map on the classifying space $B G$ of a connected Lie group $G$. The classifying space $B G$ is a Gorenstein space of dimension - $\operatorname{dim} G$; see [13] for more details. Then the result [17, Theorem 12] deduces that

$$
\operatorname{Ext}_{C^{*}\left(B G^{\times n}\right)}^{*}\left(C^{*}(B G), C^{*}\left(B G^{\times n}\right)\right) \cong H^{*-(n-1)(-\operatorname{dim} G)}(B G),
$$

where the DG right $C^{*}\left(B G^{\times n}\right)$-module structure on $C^{*}(B G)$ is induced by the diagonal map $\Delta^{(n-1)}: B G \rightarrow B G^{\times n}$. In particular, we have a generator of the vector space

$$
\operatorname{Ext}_{C^{*}\left(B G^{\times n}\right)}^{-(n-1) \operatorname{dim} G}\left(C^{*}(B G), C^{*}\left(B G^{\times n}\right)\right) \cong H^{0}(B G)=\mathbb{K},
$$

which is called a shriek map associated with the diagonal map.

Proof of Theorem 2.11. By assumption, the cohomology $H^{*}(B G)$ is a polynomial algebra, say $H^{*}(B G)=\mathbb{K}\left[x_{1}, \ldots, x_{s}\right]$. Then $H^{*}(G)$ is isomorphic to the algebra with a 2-simple system of generators $s^{-1} x_{1}, \ldots, s^{-1} x_{s}$, where $\operatorname{deg} s^{-1} x_{i}=\operatorname{deg} x_{i}-1$; see [40, page 154]. Observe that $H^{*}(G)$ is the exterior algebra generated by $s^{-1} x_{1}, \ldots$, $s^{-1} x_{s}$ if the characteristic of $\mathbb{K}$ is odd.

Claim 5.1. In the Leray-Serre spectral sequence $\left\{{ }_{L S} E_{r}^{*, *}, d_{r}\right\}$ of the fibration $G \rightarrow$ $B G^{\times(k-1)} \stackrel{B(1 \times \Delta)}{\rightarrow} B G^{\times k}$, the generators $s^{-1} x_{i}$ are transgressive. More precisely, for the transgression $\tau$, one has $\tau\left(s^{-1} x_{i}\right)=\lambda_{i}\left(x_{i} \otimes 1-1 \otimes x_{i}\right)$ for some non-zero scalar $\lambda_{i}$ under an isomorphism $H^{*}\left(B G^{\times k}\right) \cong H^{*}\left(B G^{\times(k-2)}\right) \otimes H^{*}(B G) \otimes H^{*}(B G)$.

Therefore, there is no non-trivial element in ${ }_{L S} E_{\infty}^{0 * *}$ for $*>0$. This implies that the shriek map $B(1 \times \Delta)^{!}: C^{*}\left(B G^{\times(k-1)}\right) \rightarrow C^{*-d}\left(B G^{\times k}\right)$ is a ghost map, where $d=\operatorname{dim} G$. In fact, the induced map $H^{*}\left(B(1 \times \Delta)^{!}\right)$is the integration along the fibre; see [17, Theorems 5 and 13].

We shall prove that the composition of the shriek maps $B(1 \times \Delta)^{!} \circ \cdots \circ B \Delta^{!}$: $C^{*}(B G) \rightarrow C^{*-(n-1) d}\left(B G^{\times n}\right)$ is non-trivial in $\mathrm{D}\left(C^{*}\left(B G^{\times n}\right)\right)$. To this end, we consider the homotopy pullback square

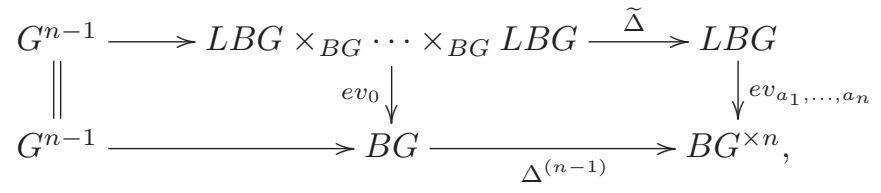

where $e v_{a_{1}, \ldots, a_{n}}$ denotes the evaluation map at points $a_{k}=\frac{k-1}{n}$ for $k=1, \ldots, n$. We regard the composite $B(1 \times \Delta) ! \circ \cdots \circ B \Delta^{!}$as the shriek map $\left(\Delta^{(n-1)}\right) !$ by choosing an appropriate orientation class of the fibration $\Delta^{(n-1)}$; see, for example, $[\mathbf{9}$, Section 2.3, Composition]. In order to show non-triviality of the shriek map $\left(\Delta^{(n-1)}\right)^{\text {! }}$, it suffices to prove that the shriek map

$$
\widetilde{\Delta}^{!}: C^{*}\left(L B G \times{ }_{B G} \cdots \times{ }_{B G} L B G\right) \rightarrow C^{*-(n-1) d}(L B G)
$$

is non-trivial since $\widetilde{\Delta}^{!}$is an extension of $\left(\Delta^{(n-1)}\right)$; ; see the proof of [17, Theorem 6$]$. We observe that $H^{*}(L B G) \cong H^{*}(B G) \otimes \Delta\left(s^{-1} x_{1}, \ldots, s^{-1} x_{s}\right)$ as an algebra. 
Let $\mathcal{K}=H^{*}(B G) \otimes \wedge\left(s^{-1} Q H^{*}(B G)\right) \otimes H^{*}(B G) \rightarrow H^{*}(B G)$ be the two sided bar resolution of $H^{*}(B G)$; see, for example, [4]. Then we have a projective resolution

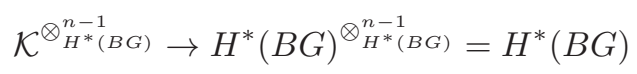

of $H^{*}(B G)$ as an $H^{*}(B G)^{\otimes n-1}$-module.

Let $\left\{E_{r}, d_{r}\right\}$ be the Eilenberg-Moore spectral sequence for the right-hand side pullback in the diagram (5.1). Computing the $E_{2}$-term by using the projective resolution $\mathcal{K}^{\otimes_{H^{*}(B G)}^{n-1}} \rightarrow H^{*}(B G)$ mentioned above, we see that

$$
\begin{aligned}
& E_{2}^{*, *} \cong H\left(H^{*}(B G)^{\otimes n} \otimes \Delta\left(s^{-1} x_{1,1}, \ldots, s^{-1} x_{1, s}, \ldots, s^{-1} x_{n-1,1}, \ldots, s^{-1} x_{n-1, s}\right)\right. \\
& \left.\otimes_{H^{*}(B G)} \otimes n H^{*}(L B G), \partial\left(s^{-1} x_{i, j}\right)=e v_{a_{1}, \ldots, a_{n}}^{*}\left(x_{i, j} \otimes 1-1 \otimes x_{i+1, j}\right)\right)
\end{aligned}
$$

as a bigraded algebra. Since $e v_{0} \simeq e v_{a_{k}}$ for any $k$, it follows that $e v_{a_{1}, \ldots, a_{n}}^{*} \circ p_{k}^{*} \simeq e v_{0}$, where $p_{k}: B G^{\times n} \rightarrow B G$ denotes the projection onto the $k$ th factor. This implies that $e v_{a_{1}, \ldots, a_{n}}^{*}\left(x_{i, j} \otimes 1-1 \otimes x_{i+1, j}\right)=0$ since $e v_{0}^{*}\left(x_{i}\right)=x_{i}$. For dimensional reasons, we see that

$$
E_{\infty}^{*, *} \cong H^{*}(L B G) \otimes \Delta\left(s^{-1} x_{1,1}, \ldots, s^{-1} x_{1, s}, \ldots, s^{-1} x_{n-1,1}, \ldots, s^{-1} x_{n-1, s}\right) .
$$

This fact enables us to conclude that the Leray-Serre spectral sequence of the upper fibration in the homotopy pull-back above collapses at the $E_{2}$-term. Therefore, it follows that the integration along the fibre $H^{*}\left((\widetilde{\Delta})^{!}\right)$is non-trivial. This completes the proof.

Proof of Claim 5.1. We consider a morphism of homotopy fibrations

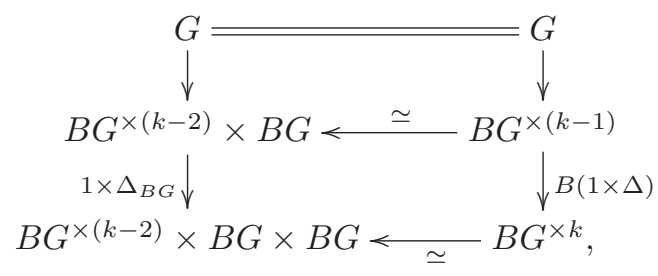

in which the horizontal maps are homotopy equivalences. Thus, in order to prove Claim 5.1, it suffices to show that the result holds for the spectral sequence of the fibration $G \rightarrow B G \stackrel{\Delta_{B G}}{\rightarrow} B G \times B G$.

Let $z_{i}: B G \rightarrow K:=K\left(\mathbb{K}, \operatorname{deg} x_{i}\right)$ be the map corresponding to the generator $x_{i}$ of $H^{*}(B G)$; that is, $z_{i}^{*}(\iota)=x_{i}$ for the fundamental class $\iota$ of $K$. In the Leray-Serre spectral sequence of the homotopy fibration $K(\mathbb{K}, \operatorname{deg} x-1) \rightarrow K \stackrel{\Delta_{K}}{\rightarrow} K \times K$, the transgression sends the fundamental class of the fibre to the element $\iota \otimes 1-1 \otimes \iota$ up to the multiplication by a non-zero scalar because $\Delta_{K}^{*}(\iota \otimes 1-1 \otimes \iota)=0$. The naturality of the morphism induced by $z_{i}$ implies that $\tau\left(s^{-1} x_{i}\right)=\lambda_{i}\left(x_{i} \otimes 1-1 \otimes x_{i}\right)$ for some non-zero scalar $\lambda_{i}$. We have the result.

Remark 5.2. The proof of Theorem 2.11 enables us to conclude that the shriek map $\left(\Delta^{(n-1)}\right) !$ is the non-trivial generator in $\operatorname{Ext}_{C^{*}\left(B G^{\times n}\right)}^{-(n-1) \operatorname{dim} G}\left(C^{*}(B G), C^{*}\left(B G^{\times n}\right)\right)$ and it is a ghost map. 
Proof of Proposition 2.12. We have a fibration of the form $G^{\times(n-1)} \rightarrow B G \stackrel{\Delta^{(n)}}{\rightarrow}$ $B G^{\times n}$. Therefore, we see that $H^{*}\left(G^{\times(n-1)}\right) \cong \operatorname{Tor}_{*}^{H^{*}\left(B G^{\times n}\right)}\left(H^{*}(B G), \mathbb{K}\right)$ and hence the torsion product is of finite dimension. Then it follows from [33, Lemma 7.1] that

$$
\operatorname{level}_{\mathrm{D}\left(C^{*}\left(B G^{\times n}\right)\right.}^{C^{*}(B G)^{\times n}}\left(C^{*}(B G)\right) \leqslant \operatorname{pd}_{H^{*}\left(B G^{\times n}\right)} H^{*}(B G)+1,
$$

where $\operatorname{pd}_{A} M$ denotes the projective dimension of an $A$-module $M$. Let

$$
\mathcal{K}^{\otimes_{H^{*}(B G)}^{n-1}} \rightarrow H^{*}(B G)^{\otimes_{H^{*}(B G)}^{n-1}}=H^{*}(B G)
$$

be the projective resolution of $H^{*}(B G)$ as an $H^{*}(B G)^{\otimes n-1}$-module introduced in the proof of Theorem 2.11. This yields that

$$
\operatorname{pd}_{H^{*}\left(B G^{\times n}\right)} H^{*}(B G) \leqslant(n-1) \operatorname{dim} Q H^{*}(B G) .
$$

We have the upper bound of the level. Proposition 2.10 and Theorem 2.11 give the lower bound.

We prove the latter half of the assertion. Since $H^{*}(B G ; \mathbb{K})$ is a polynomial algebra generated by elements with even degree, it follows from [32, Proposition 2.4] that the homotopy fibration $G^{\times(n-1)} \rightarrow B G \stackrel{\Delta^{(n)}}{\rightarrow} B G^{\times n}$ is $\mathbb{K}$-formalizable; see [33, Section 2]. Thus the result $[\mathbf{3 3}$, Proposition 5.2] implies that

$$
\text { level } C_{\mathrm{D}\left(C^{*}\left(B G^{\times n}\right)\right.}^{C^{*}(B)}\left(C^{*}(B G)\right)=(n-1) \operatorname{dim} Q H^{*}(B G ; \mathbb{K})+1 .
$$

This completes the proof.

\section{Acknowledgments}

I thank Jim Stasheff for his interest in this work and for comments on Theorem A.4. I benefited from inspiring discussions with Luc Menichi about string operations on the classifying spaces without which Theorem 2.11 could not have been obtained. I am grateful to Younggi Choi for a conversation on the proof of Claim 5.1. I also thank the referee for valuable suggestions to revise a previous version of this paper.

\section{Appendix A. A variant of Koszul duality for DG algebras}

In this section, we describe a result concerning Theorem 3.3, which is regarded as a variant of the Koszul duality for DG algebras. We also refer the reader to the paper [46] due to Positselski for a more general approach to the derived Koszul duality.

We begin by recalling the result on a coderived category due to Lefévre-Hasegawa $[38]$.

Let $\left(A, d_{A}, \varepsilon_{A}\right)$ and $\left(C, d_{C}, \varepsilon_{C}\right)$ be an augmented DG algebra and a co-augmented DG coalgebra over a field $\mathbb{K}$, respectively. By using the kernel $\bar{C}$ of the counit of $C$, we have a decomposition $C=\bar{C} \otimes \mathbb{K}$. Let $\bar{\Delta}: \bar{C} \rightarrow \bar{C} \otimes \bar{C}$ denote the reduced coproduct defined by $\bar{\Delta}(x)=\Delta(x)-x \otimes 1-1 \otimes x$. We say that a coaugmented DG coalgebra is cocomplete if $\bar{C}=\cup_{l \geqslant 1} \operatorname{Ker}\left(\bar{\Delta}^{(l)}: \bar{C} \rightarrow \bar{C}^{\otimes l+1}\right)$, where $\bar{\Delta}^{(l)}$ is the iterated coproduct defined by $\bar{\Delta}^{(l)}=\left(\bar{\Delta} \otimes 1^{\otimes l-1}\right) \circ \cdots \circ(\bar{\Delta} \otimes 1) \circ \bar{\Delta}$. By definition, a twisted 
cochain $\tau: C \rightarrow A$ is a $\mathbb{K}$-linear map of degree +1 such that $\varepsilon_{A} \circ \tau \circ \varepsilon_{C}=0$ and

$$
d_{A} \circ \tau+\tau \circ d_{C}+\mu_{A} \circ(\tau \otimes \tau) \circ \Delta_{C}=0,
$$

where $\mu_{A}$ and $\Delta_{C}$ are the multiplication of $A$ and the comultiplication of $C$, respectively. Let $M$ be a DG right $A$-module. Then we defined the twisted tensor product $M \otimes_{\tau} C$ to be the comodule $M \otimes C$ over $C$ endowed with the differential

$$
d=d_{M} \otimes 1+1 \otimes d_{C}-\left(\mu_{M} \otimes 1\right)(1 \otimes \tau \otimes 1)\left(1 \otimes \Delta_{C}\right) .
$$

For a DG $C$-comodule $N$, we define the DG module $N \otimes_{\tau} A$ similarly. Let $\Delta_{N}$ be the comodule structure of a DG $C$-comodule $N$. We say that $N$ is cocomplete if $N=\cup_{l \geqslant 1} \operatorname{Ker}\left(\bar{\Delta}_{N}^{(l)}: N \rightarrow N \otimes \bar{C}^{\otimes l}\right)$, where $\bar{\Delta}_{N}(x)=\Delta_{N}(x)-x \otimes 1$ for $x \in N$ and $\bar{\Delta}_{N}^{(l)}$ denotes the iterated comodule structure defined by the same way as the iterated coproduct on $\bar{C}$.

Let $C$ be a cocomplete DG coalgebra and $\tau_{0}: C \rightarrow \Omega C$ the canonical twisting cochain. Then the category comod $-C$ of cocomplete DG comodules over $C$ admits the structure of a model category for which $f: N \rightarrow N^{\prime}$ is a weak equivalence, by definition, if and only if $f \otimes 1: N \otimes_{\tau_{0}} \Omega C \rightarrow N^{\prime} \otimes_{\tau_{0}} \Omega C$ is a quasi-isomorphism. For the details, see [38, Théorème 2.2.2.2]. Observe that $f$ is a weak equivalence, then $f$ is a quasi-isomorphism. This fact follows form [14, Proposition 2.14]. We define the coderived category $\mathrm{D}_{c}(C)$, which is a triangulated category, to be the localization of the homotopy category of comod $-C$ with respect to the class of all weak equivalences.

Remark A.1. Let $C$ be a finite dimensional co-augmented coalgebra. The result $[\mathbf{4 2}$, 1.6.4] due to Montgomery allows one to deduce that the functor $F$ : comod-C $\rightarrow$ $C^{\vee}$-mod mentioned in Section 3 is an equivalence of categories. As mentioned above, weak equivalences between cocomplete DG-comodules are quasi-isomorphisms. Then we see that $F$ induces a functor $F_{*}: \mathrm{D}(\operatorname{comod}-C) \rightarrow D\left(C^{\vee}-\bmod \right)$ of triangulated categories. Observe that the functor $F_{*}$ is not an equivalence of triangulated categories in general. In fact, we can regard the exterior algebra $\wedge(x)$ as a Hopf algebra with a primitive element $x$ of degree -1 . Forgetting the algebra structure of $\wedge(x)$, we have a DG coalgebra $C_{1}$ endowed with the trivial differential. The argument in $[\mathbf{2 8}$, Section 4] asserts that in $\left(C_{1}\right)^{\vee}$-mod, weak equivalences form a strictly smaller class than that of quasi-isomorphisms.

On the other hand, the equivalence $F$ allows us to obtain an equivalence

$$
\widetilde{F_{*}}: \mathrm{D}_{c}(C)=\mathrm{D}(\operatorname{comod}-C) \stackrel{\simeq}{\longrightarrow} \widetilde{\mathrm{D}}\left(C^{\vee}-\bmod \right)
$$

of triangulated categories. Here $\widetilde{\mathrm{D}}\left(C^{\vee}\right.$-mod $)$ denotes the localization of the homotopy category of $C^{\vee}$-mod with respect to the class of morphisms which come from weak equivalences in comod $-C$ by $F$.

The following theorems assert that a coderived category is closely related to a derived category.

Theorem A.2 ([38, 2.2.3, Lemma 2.2.1.2, Proposition 2.2.4.1]). Let $\tau: C \rightarrow A$ be $a$ 
twisting cochain. Then one has adjoint functors

$$
\mathrm{D}_{c}(C) \underset{R:=-\otimes_{\tau} C}{\stackrel{L:=-\otimes_{\tau} A}{\longleftarrow}} \mathrm{D}(A)
$$

between triangulated categories.

Theorem A.3 ([38, Proposition 2.2.4.1]). The following are equivalent.

(i) The map $\tau$ induces a quasi-isomorphism $\Omega(C) \rightarrow A$.

(ii) The canonical map $A \otimes_{\tau} C \otimes_{\tau} A \rightarrow A$ is a quasi-isomorphism.

(iii) The functor $L$ and $R$ in Theorem A.2 are equivalences.

Let $V$ be a finite dimensional, non-negatively graded vector space with $V^{\text {odd }}=0$. Let $S V$ be the polynomial algebra and $\wedge \Sigma V$ the primitively generated coalgebra whose underlying space is the exterior algebra on $\Sigma V$. Then the projection from $\wedge \Sigma V$ to $\Sigma V$ and the inclusion form $V$ to $S V$ give rise to a twisting cochain $\tau: \wedge \Sigma V \rightarrow S V$. Thus we have exact functors between derived and coderived categories

$$
\widetilde{\mathrm{D}}\left((\wedge \Sigma V)^{\vee}-\mathrm{mod}\right) \longleftarrow \stackrel{\widetilde{F_{*}}}{\longleftarrow} \mathrm{D}_{c}(\wedge \Sigma V) \frac{L:=-\otimes_{\tau} S V}{\ll_{R:=-\otimes_{\tau} \wedge \Sigma V}} \mathrm{D}(S V) .
$$

Here $\widetilde{F_{*}}$ stands for the functor defined in Remark A.1.

The existence of the two-sided Koszul resolution (see, for example, [4]) implies that the functor $R$ gives an equivalence with inverse $L$. Indeed this follows from the equivalence of the assertions (ii) and (iii) in Theorem A.3. Moreover, since the vector space $V$ is of finite dimension, the functor $\widetilde{F_{*}}$ is also an equivalence between $\mathrm{D}(\wedge \Sigma V)$ and $\mathrm{D}\left((\wedge \Sigma V)^{\vee}\right.$-mod); see Remark A.1.

More generally, the proof of [21, Theorem 4.4] due to $\mathrm{He}$ and $\mathrm{Wu}$ enables us to deduce the following result.

Theorem A.4 (cf. [2, Theorem 7.4], [21, Theorem 4.7]). Let $A$ be a locally finite, simply-connected $D G$ algebra over a field $\mathbb{K}$. Suppose that the dual $(B A)^{\vee}$ to the bar construction is formal in the sense that $(B A)^{\vee}$ admits a TV-model $T V \stackrel{\simeq}{\rightarrow}(B A)^{\vee}$ together with a quasi-isomorphism $T V \stackrel{\widetilde{\Im}}{\rightarrow} H\left((B A)^{\vee}\right)=\operatorname{Ext}_{A}(\mathbb{K}, \mathbb{K})$. Assume further that $\operatorname{Ext}_{A}(\mathbb{K}, \mathbb{K})$ is of finite dimension. Then one has equivalences

$$
\widetilde{\mathrm{D}}\left(\operatorname{Ext}_{A}(\mathbb{K}, \mathbb{K})-\bmod \right) \underset{\frac{h}{\leftarrow}}{\stackrel{h}{\leftarrow}} \mathrm{D}(A)
$$

of triangulated categories. If $A$ is 2-connected, then $t$ satisfies the condition that $t(\mathbb{K})=\operatorname{Ext}_{A}(\mathbb{K}, \mathbb{K})^{\vee}$ and $t(A)=\mathbb{K}$ in $\widetilde{\mathrm{D}}\left(\operatorname{Ext}_{A}(\mathbb{K}, \mathbb{K})-\bmod \right)$.

Let $A$ be a 2-connected DG algebra as in Theorem A.4. Then it follows that for an object $M$ in $\mathrm{D}(A)$,

$$
\operatorname{level}_{\mathrm{D}(A)}^{A}(M)=\operatorname{level}_{\widetilde{\mathrm{D}}\left(\operatorname{Ext}_{A}(\mathbb{K}, \mathbb{K})-\bmod \right)}^{\mathbb{K}}(t(M)) .
$$

Proof of Theorem A.4. Since $A$ is simply-connected and locally finite, it follows that the bar construction is also locally finite. Thus we can assume that for the $T V$ model $T V \stackrel{\widetilde{\Im}}{\rightarrow}(B A)^{\vee}$, the graded vector space $V$ is locally finite; see the proof of 
Proposition 3.2. Then the sequence of quasi-isomorphisms

$$
E:=\operatorname{Ext}_{A}(\mathbb{K}, \mathbb{K})=H\left((B A)^{\vee}\right) \stackrel{\simeq}{\longleftarrow} T V \stackrel{\simeq}{\longrightarrow}(B A)^{\vee}
$$

of DG algebras gives rise to a sequence of quasi-isomorphisms

$$
\Omega\left(E^{\vee}\right) \stackrel{\simeq}{\longrightarrow} \Omega\left(T V^{\vee}\right) \stackrel{\simeq}{\longleftarrow} \Omega\left((B A)^{\vee \vee}\right) \stackrel{\simeq}{\longleftarrow} \Omega(B A) \stackrel{\simeq}{\longrightarrow} A
$$

as DG algebras. Thus we have equivalences

$$
\mathrm{D}\left(\Omega\left(E^{\vee}\right)\right) \underset{\beta}{\stackrel{\alpha}{\simeq}} \mathrm{D}(A)
$$

of triangulated categories for which $\beta(A)=\Omega\left(E^{\vee}\right)$ and $\beta(\mathbb{K})=\mathbb{K}$. The canonical twisting cochain $\tau_{0}: E^{\vee} \rightarrow \Omega\left(E^{\vee}\right)$ induces the identity map $\Omega\left(E^{\vee}\right) \rightarrow \Omega\left(E^{\vee}\right)$. In view of Theorem A.3, we have equivalences

$$
\mathrm{D}\left(\operatorname{comod}-\left(E^{\vee}\right)\right) \underset{R=-\otimes_{\Omega\left(E^{\vee}\right)} B\left(\Omega\left(E^{\vee}\right) ; \Omega\left(E^{\vee}\right)\right)}{\simeq} \mathrm{D}\left(\Omega\left(E^{\vee}\right)\right) .
$$

Since $E^{\vee}$ is a finite dimensional coalgebra by assumption, it follows that the functor

$$
\widetilde{F_{*}}: \mathrm{D}\left(\operatorname{comod}-\left(E^{\vee}\right)\right) \stackrel{\simeq}{\longrightarrow} \widetilde{\mathrm{D}}(E-\bmod ),
$$

which is defined in Remark A.1 gives an equivalence of triangulated categories. Then one has an equivalence $t:=\widetilde{F_{*}} \circ R \circ \beta: \mathrm{D}(A) \rightarrow \widetilde{\mathrm{D}}(E-\bmod )$.

The natural map $\sigma: E^{\vee} \rightarrow B \Omega\left(E^{\vee}\right)$ and $\eta: B\left(\Omega\left(E^{\vee}\right) ; \Omega\left(E^{\vee}\right)\right) \rightarrow \mathbb{K}$ are quasi-isomorphisms; see [14, Propositions 2.4 and 2.14]. If $A$ is 2-connected, then $E^{\vee}$ is simply-connected. Then it follows from [14, Remark 2.3] that maps $\sigma$ and $\eta$ are weak equivalences. This implies the latter half of the theorem.

The following proposition provides examples of DG algebras which satisfy the assumptions in Theorem A.4.

Proposition A.5. Let $E$ be a non-positively graded, connected DG algebra; that is, $E^{0}=\mathbb{K}$ and $E^{i}=0$ for $i>1$. Suppose further that $E$ is formal and of finite dimension. Put $A=\Omega\left(E^{\vee}\right)$. Then the algebra $(B A)^{\vee}$ is a formal and, as algebras,

$$
H\left((B A)^{\vee}\right) \cong \operatorname{Ext}_{A}(\mathbb{K}, \mathbb{K}) \cong H(E) .
$$

In consequence, the DG algebra A satisfies all the assumptions in Theorem A.4. Thus one has equivalences

$$
\widetilde{\mathrm{D}}(H(E)-\bmod ) \underset{t}{\stackrel{h}{\underset{t}{\simeq}}} \mathrm{D}\left(\Omega\left(E^{\vee}\right)\right)
$$

of triangulated categories. Assume further that $E^{\vee}$ is simply-connected. Then one has $t\left(\Omega\left(E^{\vee}\right)\right)=\mathbb{K}$ and $t(\mathbb{K})=H(E)^{\vee}$.

Proof. Since $E$ is a finite dimensional DG algebra, it follows from [14, Proposition 2.14] that there exists a quasi-isomorphism $\alpha: E^{\vee} \stackrel{\simeq}{\longrightarrow} B A=B \Omega\left(E^{\vee}\right)$ of coalge- 
bras. Let $\eta: T V \stackrel{\simeq}{\longrightarrow}\left(B \Omega\left(E^{\vee}\right)\right)^{\vee}$ be a TV-model. We then have a sequence

$$
E \cong E^{\vee \vee} \underset{\alpha^{\vee} \circ \eta}{\simeq} T V \underset{\eta}{\stackrel{\simeq}{\simeq}}(B A)^{\vee}
$$

of quasi-isomorphism of DG algebras. By assumption, the DG algebra $E$ is formal. This enables us to obtain quasi-isomorphisms $H(E) \stackrel{\simeq}{\longleftarrow} T W \stackrel{\simeq}{\longrightarrow} E$. The lifting lemma [15, Lemma 3.6] yields a quasi-isomorphism $T V \stackrel{\simeq}{\longrightarrow} H(E)$ of DG algebras and hence $(B A)^{\vee}$ is formal.

Example A.6. Let $E$ be an exterior algebra $\wedge\left(x_{1}, \ldots, x_{n}\right)$ generated by $x_{1}, \ldots, x_{n}$, where $-\operatorname{deg} x_{i}$ is odd for any $i$. We have an isomorphism $H\left(\Omega\left(E^{\vee}\right)\right) \cong H\left((B E)^{\vee}\right)=$ $\operatorname{Tor}_{E}(\mathbb{K}, \mathbb{K})^{\vee}$ of algebras which sends the cycles $\left\langle x_{i}^{\vee}\right\rangle$ to $\left[x_{i}\right]^{\vee}$. Moreover, there exists an isomorphism

$$
\eta: \operatorname{Tor}_{E}(\mathbb{K}, \mathbb{K})=H(B E) \stackrel{\cong}{\rightrightarrows} \Gamma\left[s x_{1}, \ldots, s x_{n}\right]
$$

of coalgebras such that $\eta\left(\left[x_{i}\right]\right)=s x_{i}$, where $\operatorname{deg} s x_{i}=\operatorname{deg} x_{i}-1$ and $\Gamma\left[s x_{1}, \ldots, s x_{n}\right]$ stands for the divided power Hopf algebra with the comultiplication $\Delta\left(\gamma_{i}\left(s x_{j}\right)\right)=$ $\sum_{k+l=j} \gamma_{i}\left(s x_{j}\right) \otimes \gamma_{k}\left(s x_{j}\right)$; see the proof of [31, Lemma 1.5]. Thus we see that the algebra $H\left(\Omega\left(E^{\vee}\right)\right)$ is isomorphic to the polynomial algebra $\mathbb{K}\left[s x_{1}^{\vee}, \ldots, s x_{n}^{\vee}\right]$, where $\operatorname{deg} s x_{i}^{\vee}=-\operatorname{deg} x_{i}+1$. Since the algebra $\Omega\left(E^{\vee}\right)$ is free, it follows that there exists a quasi-isomorphism $\theta: \Omega\left(E^{\vee}\right) \stackrel{\widetilde{\sim}}{\rightarrow} \mathbb{K}\left[s x_{1}^{\vee}, \ldots, s x_{n}^{\vee}\right]$ of algebras such that $\theta\left(\left\langle x_{i}^{\vee}\right\rangle\right)=s x_{i}^{\vee}$ for $i$. This implies that $\Omega\left(E^{\vee}\right)$ is formal. Therefore, Theorem A.4 and Proposition A.5 enable us to obtain equivalences

$$
\widetilde{\mathrm{D}}\left(\wedge\left(x_{1}, \ldots, x_{n}\right)-\bmod \right) \underset{\frac{h}{\underset{t}{\simeq}}}{\stackrel{\stackrel{\simeq}{\leftarrow}}{\leftarrow}} \mathrm{D}\left(\mathbb{K}\left[s x_{1}^{\vee}, \ldots, s x_{n}^{\vee}\right]\right)
$$

of triangulated categories. This result is a variant of [2, Theorem 7.4]; see also [28, Section 4].

\section{References}

[1] J.F. Adams, On the cobar construction, Proc. Natl. Acad. Sci. USA 42 (1956), 409-412.

[2] L.L. Avramov, R.-O. Buchweitz, S.B. Iyengar and C. Miller, Homology of perfect complexes, Adv. Math. 223 (2010), 1731-1781. arXiv:math.AC/ 0609008v2.

[3] L.L. Abramov and S.B. Iyengar, Cohomology over Complete Intersections via Exterior Algebras, London Math. Soc. Lecture Note Ser. 375, Cambridge University Press, Cambridge, 2010, pp. 52-75.

[4] P.F. Baum and L. Smith, Real cohomology of differential Fibre bundles, Comment. Math. Helv. 42 (1967), 171-179.

[5] D.J. Benson, S.B. Iyengar and H. Krause, Stratifying triangulated categories, J. Topol. 4 (2011), 641-666.

[6] A.J. Blumberg, R.L. Cohen and C. Teleman, Open-closed field theories, string topology, and Hochschild homology, in: Alpine Perspectives on Algebraic Topology, Contemp. Math. 504. Amer. Math. Soc., Providence, RI, 2009, pp. 53-76. 
[7] A. Bondal and M. Ban den Bergh, Generators and representability of functors in commutative and non-commutative geometry, Mosc. Math. J. 3 (2003), 1-36.

[8] M. Chas and D. Sullivan, String topology, arXiv:math.GT/0107187.

[9] D. Chataur and L. Menichi, String topology of classifying spaces, J. Reine Angew. Math. 669 (2012), 1-45.

[10] J.D. Christensen, Ideals in triangulated categories: Phantoms, ghosts and skeleta, Adv. Math. 136 (1998) 284-339.

[11] W. Dwyer and J.P.C. Greenlees, Complete modules and torsion modules, Amer. J. Math. 124 (2002), 199-220.

[12] W.G. Dwyer, J.P.C. Greenlees and S. Iyengar, Duality in algebra and topology. Adv. Math. 200 (2006), 357-402.

[13] Y. Félix, S. Halperin and J.-C. Thomas, Gorenstein spaces. Adv. Math. 71 (1988), 92-112.

[14] Y. Félix, S. Halperin and J.-C. Thomas, Adams' cobar equivalence, Trans. Amer. Math. Soc. 329 (1992), 531-549.

[15] Y. Félix, S. Halperin and J.-C. Thomas, Differential graded algebras in topology, in: I.M. James (Ed.), Handbook of Algebraic Topology, Elsevier, Amsterdam, 1995, pp. 829-865.

[16] Y. Félix, S. Halperin and J.-C. Thomas, Rational Homotopy Theory, Grad. Texts in Math. 205, Springer-Verlag.

[17] Y. Félix and J.-C. Thomas, String topology on Gorenstein spaces, Math. Ann. 345 (2009), 417-452.

[18] A. Frankild and P. Jørgensen, Homological properties of cochain differential graded algebras, J. Algebra 320 (2008), 3311-3326.

[19] V.K.A.M. Gugenheim and J.P. May, On the theory and applications of differential torsion products, Mem. Amer. Math. Soc. 142 (1974).

[20] S. Halperin and J.-M. Lemaire, Notions of category in differential algebra, in: Algebraic Topology: Rational Homotopy, Lecture Notes in Math. 1318, Springer, Berlin, New York, 1988, pp. 138-154.

[21] J.-W. He and Q.-S. Wu, Koszul differential graded algebras and BGG correspondence, J. Algebra 320 (2008), 2434-2962.

[22] M. Hovey and K. Lockridge, The ghost dimension of a ring, Proc. Amer. Math. Soc. 137 (2009), 1907-1913.

[23] D. Husemoller, J.C. Moore and J. Stasheff, Differential homological algebra and homogeneous spaces. J. Pure Appl. Algebra 5 (1974), 113-185.

[24] P. Jørgensen, Auslander-Reiten theory over topological spaces, Comment. Math. Helv. 79 (2004), 160-182.

[25] P. Jørgensen, The Auslander-Reiten quiver of a Poincaré duality space, Algebr. Represent. Theory 9 (2006), 323-336.

[26] P. Jørgensen, Calabi-Yau categories and Poincaré duality spaces, in: Trends in Representation Theory of Algebras and Related Topics. EMS Ser. Congr. Rep., Bur. Math. Soc., Zürich, 2008, pp. 399-431. 
[27] B. Keller, Deriving DG categories, Ann. Sci. Éc. Norm. Supér. 27 (1994), 63-102.

[28] B. Keller, Koszul duality and coderived categories, preprint, 2003.

[29] A. Kono and K. Kuribayashi, Module derivations and cohomological splitting of adjoint bundles, Fund. Math. 180 (2003), 199-221.

[30] I. Kriz and J.P. May, Operads, algebras, modules and motives, Astérisque 233 (1995).

[31] K. Kuribayashi, On the mod $p$ cohomology of spaces of free loops on the Grassmann and Stiefel manifolds, J. Math. Soc. Japan 43 (1991), 331-346.

[32] K. Kuribayashi, On the levels of maps and topological realization of objects in a triangulated category, J. Pure Appl. Algebra 216 (2012), 752-765.

[33] K. Kuribayashi, Upper and lower bounds of the (co)chain type level of a space, Algebr. Represent. Theory 16 (2013), 129-153.

[34] K. Kuribayashi and L. Menichi, The BV-algebra in string topology of classifying spaces, in preparation.

[35] K. Kuribayashi, L. Menichi and T. Naito, Remarks on derived string topology, in preparation.

[36] K. Kuribayashi, L. Menichi and T. Naito, Behavior of the Eilenberg-Moore spectral sequence in derived string topology, Topology Appl. 164 (2014), 24-44.

[37] K. Kuribayashi, L. Menichi and T. Naito, Derived string topology and the Eilenberg-Moore spectral sequence, Israel J. Math. 209 (2015), 745-802.

[38] K. Lefèvre-Hasegawa, Sur les $A_{\infty}$-catégories, Thèse de Doctorat, Université Denis Diderot-Paris 7, November, 2003, arXiv: math.CT/0310337v1.

[39] X.F. Mao, Ghost length, cone length and complete level of DG modules, Acta Math. Sin. (Engl. Ser.) 29 (2013), 1279-1310.

[40] J. McCleary, A User's Guide to Spectral Sequences, second edition, Cambridge Stud. Adv. Math. 58. Cambridge University Press, Cambridge, 2001.

[41] M. Mimura and H. Toda, Topology of Lie Groups, I, II, translated from the 1978 Japanese edition by the authors, Transl. Math. Monogr. 91. American Mathematical Society, Providence, RI, 1991.

[42] S. Montgomery, Hopf Algebras and Their Actions on Rings, CBMS Reg. Conf. Ser. Math. 82, American Mathematical Society, Providence, RI, 1993.

[43] H.J. Munkholm, The Eilenberg-Moore spectral sequence and strongly homotopy multiplicative maps, J. Pure Appl. Algebra 5 (1974), 1-50.

[44] A. Murillo, The virtual Spivak fiber, duality on fibrations and Gorenstein spaces, Trans. Amer. Math. Soc. 359 (2007), 3577-3587.

[45] J. Neisendorfer, Algebraic Methods in Unstable Homotopy Theory, New Math. Monogr. 12, Cambridge University Press, Cambridge, 2010.

[46] L. Positselski, Two kinds of derived categories, Koszul duality, and comodulecontramodule correspondence, Mem. Amer. Math. Soc. 212 (2011).

[47] R. Rouquier, Dimensions of triangulated categories, J. K-Theory 1 (2008), 193-256. 
[48] K. Schmidt, Auslander-Reiten theory for simply connected differential graded algebras, preprint (2008), arXiv:math.RT/0801.0651v1.

[49] S. Shamir, On the string topology category of compact Lie groups, Adv. Math. 261 (2014), 122-153.

Katsuhiko Kuribayashi kuri@math.shinshu-u.ac.jp

Department of Mathematical Sciences, Faculty of Science, Shinshu University, Matsumoto, Nagano 390-8621, Japan 